ARTICLE

DOI: $10.1038 /$ s41467-018-07171-4

\title{
Active intermixing of indirect and direct neurons builds the striatal mosaic
}

\author{
Andrea Tinterri ${ }^{1,2,3}$, Fabien Menardy (10 4 , Marco A. Diana ${ }^{5}$, Ludmilla Lokmane (i) ${ }^{1}$, Maryama Keita', \\ Fanny Coulpier ${ }^{6}$, Sophie Lemoine ${ }^{6}$, Caroline Mailhes ${ }^{7}$, Benjamin Mathieu ${ }^{8}$, Paloma Merchan-Sala ${ }^{9}$, \\ Kenneth Campbell ${ }^{9}$, Ildiko Gyory ${ }^{10}$, Rudolf Grosschedl ${ }^{10}$, Daniela Popa ${ }^{4}$ \& Sonia Garel (i) ${ }^{1}$
}

The striatum controls behaviors via the activity of direct and indirect pathway projection neurons (dSPN and iSPN) that are intermingled in all compartments. While such cellular mosaic ensures the balanced activity of the two pathways, its developmental origin and pattern remains largely unknown. Here, we show that both SPN populations are specified embryonically and intermix progressively through multidirectional iSPN migration. Using conditional mutant mice, we found that inactivation of the dSPN-specific transcription factor Ebf1 impairs selective dSPN properties, including axon pathfinding, while molecular and functional features of iSPN were preserved. Ebf1 mutation disrupted iSPN/dSPN intermixing, resulting in an uneven distribution. Such architectural defect was selective of the matrix compartment, highlighting that intermixing is a parallel process to compartment formation. Our study reveals while iSPN/dSPN specification is largely independent, their intermingling emerges from an active migration of iSPN, thereby providing a novel framework for the building of striatal architecture.

\footnotetext{
${ }^{1}$ Brain Development and Plasticity Team, Institut de Biologie de I'Ecole Normale Supérieure (IBENS), Ecole Normale Supérieure, CNRS, INSERM, PSL Université Paris, 75005 Paris, France. ${ }^{2}$ Boehringer Ingelheim Fonds, Foundation for Basic Research in Medicine, Schusterstr. 46-48, 55116 Mainz, Germany. ${ }^{3}$ Ecole de Neurosciences de Paris lle de France, 15 rue de I'Ecole de Medicine, 75006 Paris, France. ${ }^{4}$ Neurophysiology of Brain Circuits Team, Institut de Biologie de I'Ecole Normale Supérieure (IBENS), Ecole Normale Supérieure, CNRS, INSERM, PSL Université Paris, 75005 Paris, France. ${ }^{5}$ UPMC University Paris 06, UM119 Neuroscience Paris Seine (NPS), CNRS UMR8246, Inserm, U1130 Paris, France. ${ }^{6}$ Genomics Facility, Institut de Biologie de l'Ecole Normale Supérieure (IBENS), Ecole Normale Supérieure, CNRS, INSERM, PSL Université Paris, 75005 Paris, France. ${ }^{7}$ Acute Transgenesis Facility, Institut de Biologie de l'Ecole Normale Supérieure (IBENS), Ecole Normale Supérieure, CNRS, INSERM, PSL Université Paris, 75005 Paris, France. ${ }^{8}$ Photon Microscopy Facility, Institut de Biologie de l'Ecole Normale Supérieure (IBENS), Ecole Normale Supérieure, CNRS, INSERM, PSL Université Paris, 75005 Paris, France. ${ }^{9}$ Division of Developmental Biology, Cincinnati Children's Hospital Medical Center, Cincinnati, OH 45229, USA. ${ }^{10}$ Department of Cellular and Molecular Immunology, Max Plank Institute of Immunobiology, Stuebeweg 51, 79108 Freiburg, Germany. These authors contributed equally: Fabien Menardy and Marco A. Diana. Correspondence and requests for materials should be addressed to S.G. (email: garel@biologie.ens.fr)
} 
T he dorsal striatum controls major brain functions, such as motor behaviors and habit formation, through the coordinated activation of descending direct and indirect pathways. Consistently, loss or damage of striatal projection neurons (SPN) is associated with a spectrum of pathologies that include Parkinson's disease, Huntington's disease and obsessivecompulsive disorders. SPN are medium-spiny GABAergic neurons that account for $90-95 \%$ of striatal neurons and are divided into two subtypes: (i) direct SPN (dSPN), which project to the substantia nigra (SN), express the D1 dopamine receptor and the neuropeptide Substance P; (ii) indirect SPN (iSPN), which send axons to the globus pallidus (GP), express D2 dopamine receptor and the opioid peptide Enkephalin. Functionally, it is proposed that dSPN activation promotes action selection and positive reinforcement, whereas iSPN are important to suppress unwanted motor sequences ${ }^{1-4}$. dSPN and iSPN are completely intermixed within the striatum, thereby forming a relatively uniform mosaic ${ }^{5,6}$. Building on this relatively simple cellular organization, the dorsal striatum is split into at least two major compartments, cellular islands known as striosomes and a surrounding matrix, which form immunohistologically and functionally distinct modules differing by their input and output patterns of connectivity ${ }^{7-11}$. In such precise three-dimensional organization, the intermingling of dSPN and iSPN ensures the balanced activation of direct and indirect pathways.

While functional studies revealed distinct roles for dSPN/iSPN and striosomes/matrix, much less is known on how they develop. SPN are generated in the embryonic lateral ganglionic eminence (LGE) and migrate radially to form the striatum ${ }^{12-17}$. Both SPN subtypes are present in the entire striatum, except for a restricted caudal domain ${ }^{5}$, and several transcription factors have been involved in their generation and differentiation including Ctip2, FoxP1, and FoxP2 ${ }^{18-23}$. In addition, distinct transcriptomic programs have been involved in the specification of either dSPN or iSPN, including Isletl and Ebf1 for dSPN, or Sp9 and Six 3 for iSPN $^{16,24-29}$. In particular, the conditional inactivation of Islet 1 impairs the differentiation of early-born $\mathrm{dSPN}^{24,28}$ and the full inactivation of $E b f 1$ leads to a defective development of late-born dSPN, disorganized projections to the $\mathrm{SN}$ and altered survival of matrix dSPN at postnatal stages ${ }^{25-27}$. Regardless of their subtype, early progenitors give rise to striosome SPNs whereas laterderived progenitors generate matrix SPN, which delineate the two compartments potentially via migration and selective cellsorting ${ }^{11,17,30-33}$. In sharp contrast, how dSPN and iSPN are specified and integrated into striatal circuits remains largely to be characterized.

Here we show that formation of the striatal mosaic relies on the early specification of SPN subtypes combined with a dSPNdependent tangential migration of iSPN. We found that dSPN and iSPN form two molecularly defined populations throughout development, which only progressively intersperse. Using time-lapse imaging, we observed that iSPN undergo multidirectional migration, thereby intermixing with dSPN. Furthermore, we took advantage of a unique combination of genetic tools, including embryonic in vivo fate map and conditional knockout models, to examine the role of Ebf1 in the differentiation of dSPN and striatal mosaic formation. We found that $E b f 1$ deletion in dSPN (i) perturbed specific aspects of direct neurons differentiation, leading to defective axogenesis and integration in cortico-striatal circuits, without affecting iSPN cardinal properties (ii) impaired intermixing of dSPN and iSPN in the matrix. These results establish Ebf1 as a master regulator of dSPN connectivity and intermixing with iSPN in the matrix compartment. Furthermore, our study reveals that the active intermingling of early-specified dSPN and iSPN is required to assemble the striatal mosaic.

\section{Results}

Defining a molecular fingerprint of embryonic dorsal dSPN. To investigate how dSPN and iSPN are specified, positioned and assembled in the striatum, we searched for specific markers of these two neuronal populations during embryogenesis. To this aim, we took advantage of the BAC transgenic mouse line Drd2$E G F P^{34}$, which has been widely used to label iSPN in neonates and is expressed embryonically $\mathrm{y}^{35,36}$. In addition, we crossed $R 26^{\mathrm{mt} /}$ ${ }^{m t}$ reporter mice ${ }^{37}$ with Islet $1^{\text {Cre }}$ transgenic mice, which mediate Cre-recombination in dSPN from embryonic stages ${ }^{24,28}$. We first confirmed that these two distinct transgenic lines respectively labeled iSPN and dSPN at postnatal day (P) 5, when the two populations can be unambiguously defined ${ }^{35}$. In Islet $1^{\mathrm{Cre}} ; \mathrm{R}^{2} \mathrm{6}^{\mathrm{mt} / \mathrm{+}}$; Drd2-EGFP mice, we found that tdTomato-positive $\left(\right.$ tom $\left.^{+}\right)$cells constituted approximately half of all Ctip $2^{+} \mathrm{SPN}^{18}$ in both striosome and matrix compartments (as defined in Supplementary Fig. 1) and were largely unlabeled by $\operatorname{Drd2-EGFP~(Fig.~1a-d).~}$ Using these two mouse lines, we found that iSPN and dSPN lineages are non-overlapping throughout embryogenesis, consistently with previous studies focusing on SPN subtypes specification ${ }^{16,24,26-29}$. We furthermore identified high expression of transcription factors FoxP2 and Ebf1 as specific of dSPN at E13.5 and E17.5 (Fig. 1e-h). These results identify a dSPN developmental fingerprint which, combined to the iSPN-specific Drd2EGFP expression, delineates two segregated populations of Ctip2 ${ }^{+} /$Foxp $1^{+}$SPN throughout development (Fig. 1i). Our findings thus indicate that dSPN and iSPN exhibit distinct molecular identities from the earliest steps of striatogenesis and provides us with tools to follow their integration in the striatal architecture.

iSPN progressively intermix with dSPN via intrastriatal migration. We took advantage of the early molecular fingerprints to monitor the dynamics of SPN distribution and thereby extend our comprehension of how the two populations are specified and assembled (Fig. 2). Previous studies showed that all SPN are generated in the LGE over an extended period of time and migrate radially to form the striatum ${ }^{12-15,17}$. Moreover, birthdating experiments indicate that a majority of earliest-born SPN are $\mathrm{dSPN}^{17,30}$. Consistently, we found that the striatum at E12.5 contained mostly dSPN neurons and few scattered $\mathrm{GFP}^{+}$iSPN (Fig. 2a, b). GFP + iSPN were detected in larger numbers at E13.5 and E14.5, albeit concentrated in the lateral striatum (Fig. 2c-h), which contains early-born neurons that will mostly contribute to striosomes $^{14,38}$. From E15.5 onwards, we detected a progressively increasing number of $\mathrm{GFP}^{+}$iSPN in the initially dSPN-dense part of the medial striatum (Fig. $2 \mathrm{i}-\mathrm{n}$ ). Thus iSPN generated in the LGE proliferative zones intersperse into dSPN-dense territories, first in the lateral striatum and then medially, in a gradual process that spans several days (Fig. 20). This phenomenon was temporally and spatially overlapping with the formation of striosome and matrix compartments $14,15,17,31$.

Such progressive emergence of Drd2-EGFP ${ }^{+}$cells in $\mathrm{dSPN}$ dense territories could be explained by: (i) delayed expression of Drd2-EGFP compared to dSPN markers; (ii) sequential generation of dSPN and iSPN for each territory; (iii) tangential dispersion of iSPN in the striatal mantle. To discriminate between these non-exclusive possibilities, we first examined whether immature iSPN in the LGE subventricular zone (SVZ) expressed Drd2-EGFP and whether timed EdU injections showed preferential labeling of either iSPN or dSPN. We found that immature iSPN already expressed Drd2-EGFP in the SVZ (Supplementary Fig. 2a-b) and that both iSPN and dSPN neurons were produced continuously from E11 onwards (Supplementary Fig. 2c-e), consistently with previous studies ${ }^{17,36}$. iSPN, however, constitute a minority of the early-born SPN, as revealed by EdU 

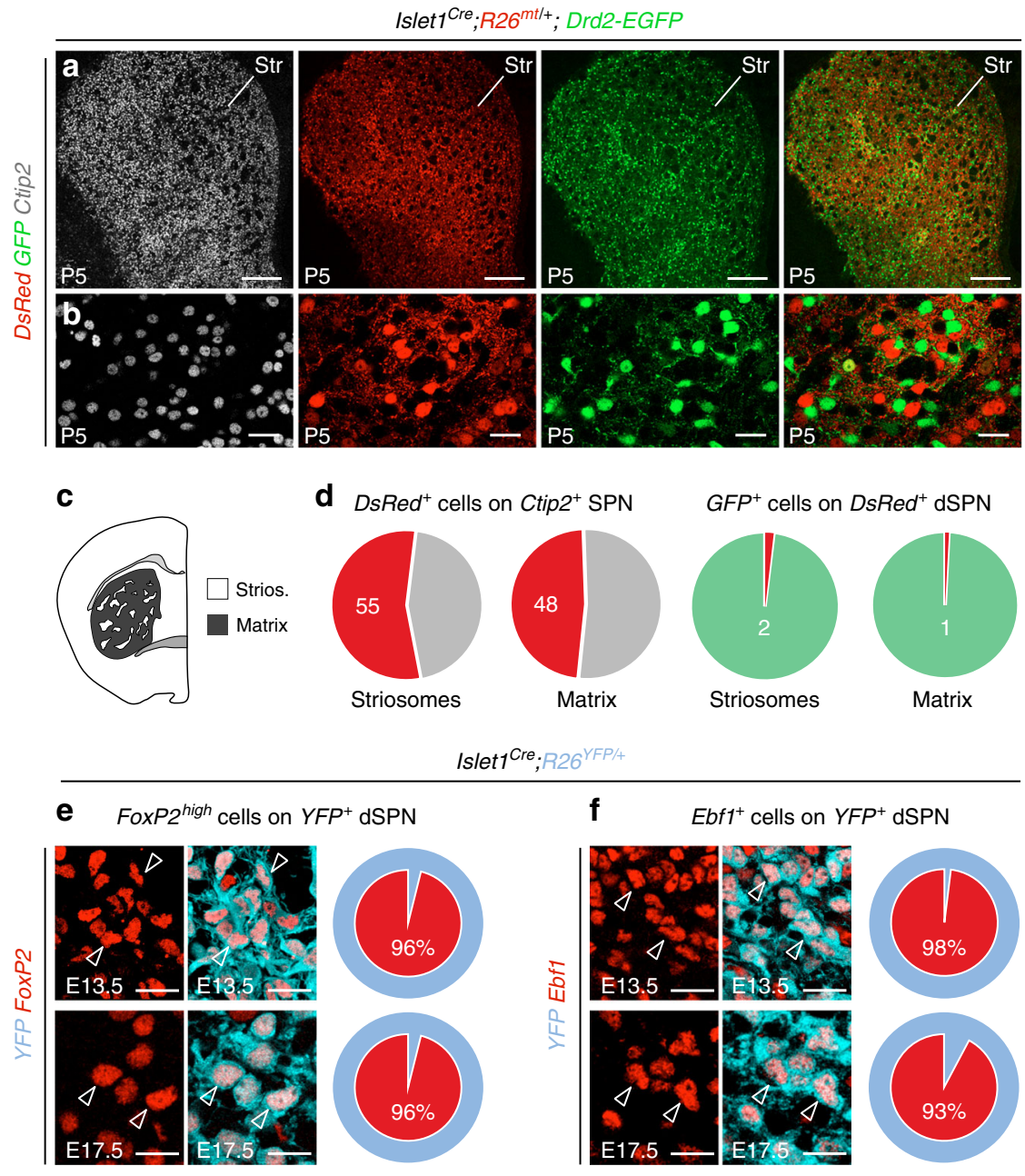

Drd2-EGFP

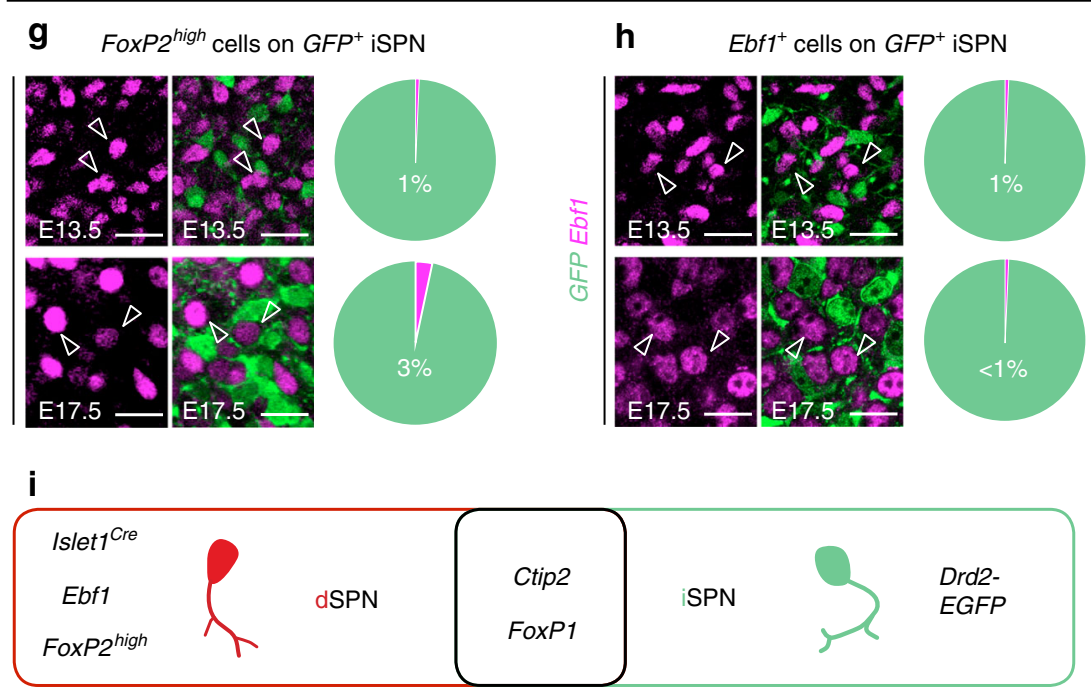

Fig. 1 Definition of an embryonic molecular fingerprint of dSPN. a, b Coronal section of P5 Islet $7^{C r e} ; R 26^{m t /+} ;$ Drd2-EGFP mice showing Ctip2-immunostaining in all SPN and non-overlapping labeling of tdTomato in dSPN and GFP in iSPN in the dorsal striatum (Str). c Schematic representation of striatal compartments. d Quantification of the percentage of tdTomato ${ }^{+} \mathrm{dSPN}$ in striatal compartments $\left(n=3\right.$ mice), on the total of Ctip2 ${ }^{+}$cells ( $55 \pm 2 \%$ in Strios., $48 \pm 7 \%$ in matrix) and on the total of GFP + cells ( $2 \pm 1 \%$ in Strios., $1 \pm 1 \%$ in matrix). e, $\mathbf{f}$ Co-immunostaining for FoxP2 ${ }^{\text {high }}$ and Ebf1 labels the YFPpositive dSPN lineage in Islet7Cre;R26 YFP/+ embryos at both E13.5 (96 $\pm 1 \%$ for Foxp2 ${ }^{\text {high; }} ; 98 \pm 1 \%$ for Ebf1) and E17.5 (96 $\pm 2 \%$ for Foxp2 ${ }^{\text {high; }}$; $93 \% \pm 1 \%$ for Ebf1) ( $n=3$ mice for each stage). $\mathbf{g}$, h Conversely, FoxP2 and Ebf1 are scarcely co-expressed by Drd2-EGFP + cells at E13.5 ( $1 \pm 1 \%$ and $1 \pm 0,1 \%$ of GFP + cells, respectively) and E17.5 ( $3 \pm 3 \%$ and $1 \pm 0,1 \%$ of GFP ${ }^{+}$cells, respectively) ( $n=3$ mice for each stage). $\mathbf{i}$ Schematic representation of the embryonic genes common and specific to dSPN and iSPN. Results are presented as mean values \pm sem. Scale bars equal $250 \mu \mathrm{m}(\mathbf{a})$, and $25 \mu \mathrm{m}(\mathbf{b}, \mathbf{e}-\mathbf{h})$. Str striatum, Strios. Striosomes 


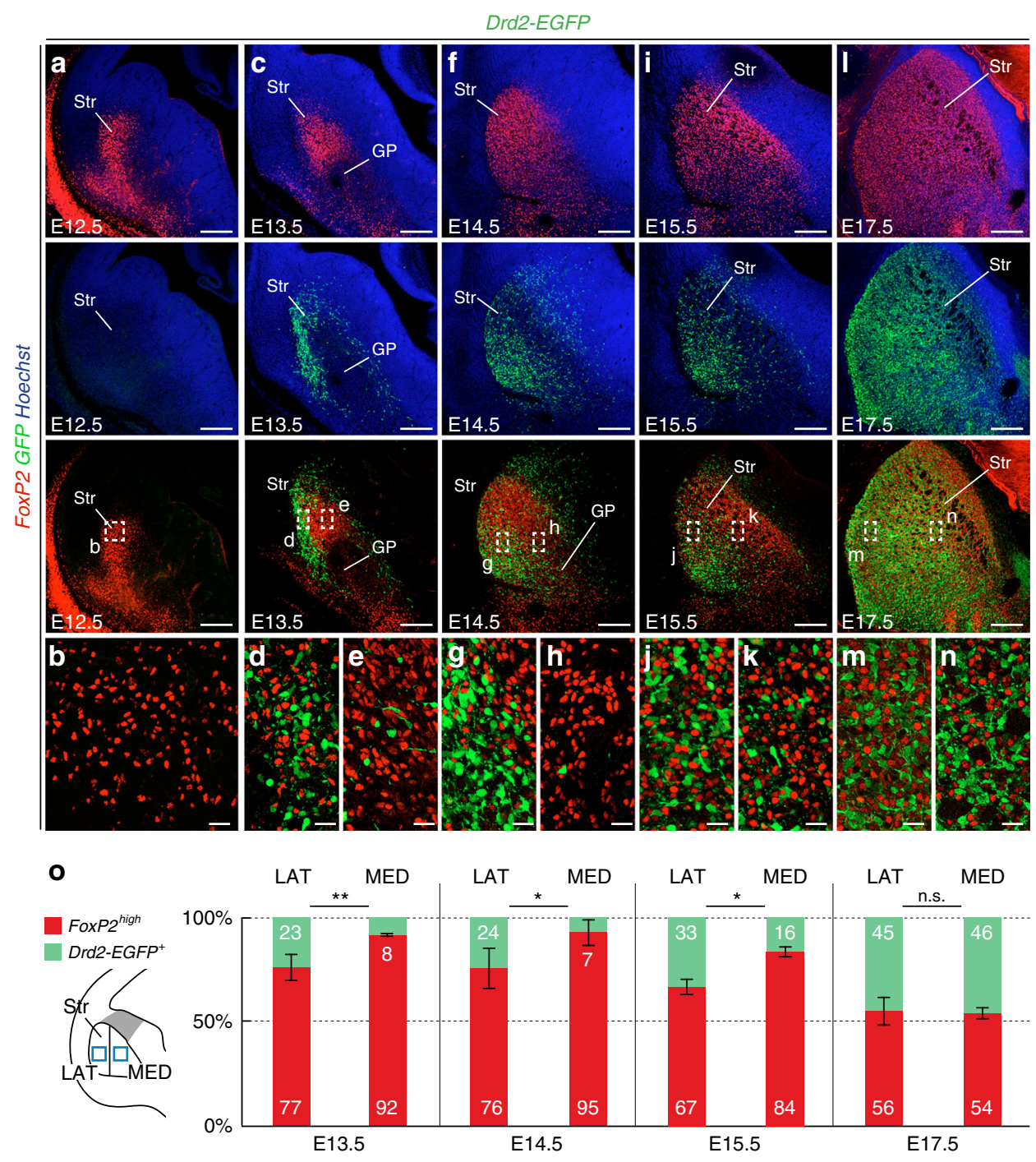

Fig. 2 Progressive insertion of iSPN into the dSPN-dense striatal mantle. a, b At E12.5, only FoxP2 ${ }^{+}$dSPN are located in the striatal mantle, while Drd2-EGFP + iSPN cannot be detected (inset in b). c-e At E13.5, Drd2-EGFP+ iSPN are mostly located in the lateral mantle (inset in d, $23 \pm 6 \%$ of total number of Drd2EGFP $^{+}$and FoxP2 ${ }^{+}$cells) while almost none are detected medially (inset in $\mathbf{e}, 8 \pm 1 \%, p=0.008$ ). $\mathbf{f}-\mathbf{h}$ At E14.5, Drd2-EGFP+ cells account for $24 \pm 10 \%$ of all SPN laterally, but only $7 \pm 6 \%$ medially (insets in $\mathbf{g}$ and $\mathbf{h}, p=0.02$ ). i-k At E15.5, more iSPN are found in the medial part of the striatum, but the distribution is still significantly different (insets in $\mathbf{j}$ and $\mathbf{k}, 33 \pm 3 \%$ laterally versus $16 \pm 4 \%$ medially, $p=0.01$ ). I-n At E17.5, iSPN are distributed similarly in the lateral and medial striatum (insets in $\mathbf{m}$ and $\mathbf{n}, 45 \pm 7 \%$ laterally versus $46 \pm 2 \%$ medially, $p>0.05$ ), showing that iSPN progressively achieve a homogeneous distribution in the striatum. 0 Quantifications of the dSPN Foxp2 ${ }^{\text {high }}$ /iSPN GFP+ distribution (at least $n=3$ mice for each stage). Results are presented as mean \pm standard deviation. Two-tailed non-parametric Mann-Whitney $U$ test was used for statistical comparison; ${ }^{\star} p$-value $<0.05$, ${ }^{\star \star} p$-value $<0.01$. Scale bars equal $200 \mu \mathrm{m}(\mathbf{a}, \mathbf{c}, \mathbf{f}, \mathbf{I}, \mathbf{i})$ and $25 \mu \mathrm{m}(\mathbf{b}, \mathbf{d}, \mathbf{e}, \mathbf{g}, \mathbf{h}, \mathbf{j}, \mathbf{k}, \mathbf{m}, \mathbf{n})$. GP globus pallidus, LAT lateral striatum, MED medial striatum, Str striatum

staining (Supplementary Fig. 2c-e) as well as labeling with the dSPN-specific Foxp2 immunostaining and Islet $1^{\text {Cre }}$-driven recombination (Supplementary Fig. 2f-h). Taken together, our results indicate that Drd2-EGFP labels iSPN as they differentiate and enter the striatum primordium.

It is thus unlikely that mosaic formation is entirely due to either a delay in Drd2-EGFP expression or a sequential production of SPN subtypes. To examine whether iSPN disperse tangentially inside the striatum, we performed two-photon timelapse imaging on Drd2-EGFP embryonic slices ${ }^{39}$. We found that GFP + iSPN show multidirectional saltatory migration within the striatal mantle at E15.5 (Fig. 3a-e; Movie 1). By performing cell behavior analyses over several time-lapse acquisitions, we found that iSPN have a global displacement away from the SVZ but present multidirectional trajectories inside the striatum (Fig. $3 \mathrm{f}-\mathrm{i}$ and Supplementary Fig. 3). In particular, iSPN changed their migration direction over time (Movie 1). Importantly, we confirmed that Drd2-GFP ${ }^{+}$cells in slices are Ctip2 ${ }^{+}$SPN and that the processes of these neurons also present multiple orientations in fixed embryonic tissue (Supplementary Fig. 3). Thus, in contrast to the assumption that SPN only migrate radially, iSPN undergo a tangential migration within the striatum and intermix with dSPN.

Taken together, our work shows that dSPN and iSPN form segregated embryonic populations that intermix over time, first in the lateral early-born proto-striosomes and then in the medial region. This process occurs at least in part via a multidirectional migration of iSPN thereby raising questions about the underlying 


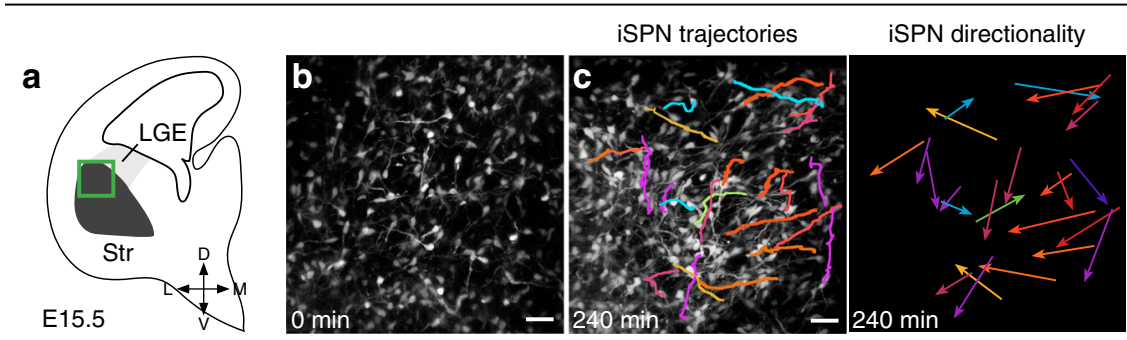

d

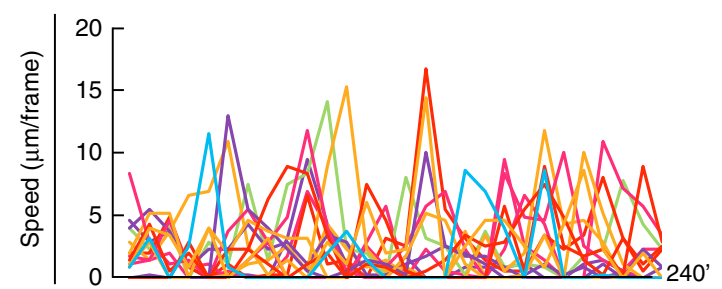

$\mathbf{f}$
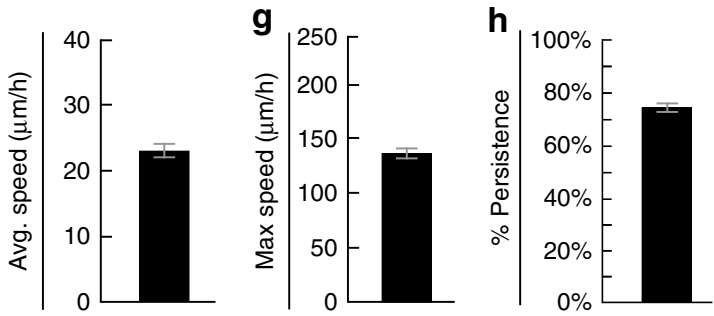

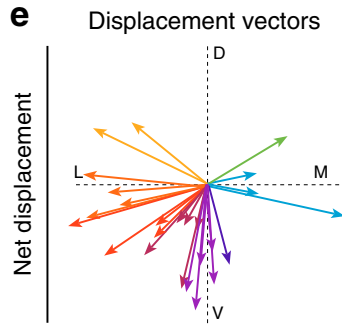

Fig. 3 Tangential migration of iSPN in the striatal mantle. a Schematic coronal hemisection of a Drd2-EGFP + slice illustrating the area where multi-photon time-lapse imaging was performed. b-e Sample image (b, c with superimposed trajectories of individual cells) from a video tracking of iSPN migration allowing the identification of cell trajectory and directionality (c), saltatory behavior (d), and multidirectional displacement vectors (e; colors illustrate directionality). $\mathbf{f}-\mathbf{i}$ Analysis of all tracked iSPN ( $n=61$ cells, $n=4$ independent experiments) shows a moderate average net speed (f, $21.3 \pm 1 \mu \mathrm{m} / \mathrm{h}$ ), a high maximum speed ( $\mathbf{g}, 124.8 \pm 5 \mu \mathrm{m} / \mathrm{h}$ ) and strong persistence (h, $74 \pm 15 \%$, ratio between net displacement speed and total distance traveled). i Analysis of mean directionality shows that iSPN migrate tangentially within the striatal mantle (color code similar to $\mathbf{c}$ and $\mathbf{e}$ ). Results are presented as mean \pm s.e.m. Scale bar equals $30 \mu \mathrm{m}$. D dorsal, L lateral, LGE lateral ganglionic eminence, M medial, Str striatum, V ventral

mechanisms and possible interactions between the two populations.

Ebf1 conditional deletion affects specific aspects of dSPN differentiation. To address these issues, we searched for mouse models selectively perturbing the development of one of the SPN subtypes. Transcription factors specifically involved in either dSPN or iSPN differentiation include Islet1 and Ebf1 or Sp9 and Six3, respectively ${ }^{16,24-29}$. In contrast to Islet1, Six3, or Sp9 deletions, which induce cell death in the targeted population ${ }^{16,24,28,29}$, Ebf1 inactivation has been previously proposed to impair dSPN wiring in the striatal matrix ${ }^{25-27}$. We thus generated and compared two different conditional Ebf1 mutants (cKO), using the dSPN-restricted Islet $1^{C r e}$ line and the Dlx5/6::Cre. Indeed, Islet $1^{\text {Cre }}$ drives recombination in $\mathrm{dSPN}$ and cholinergic interneurons ${ }^{24,28,40}$ and $D l x 5 / 6::$ Cre recombines in all SPN and all interneurons ${ }^{41-44}$. Importantly, Ebf1 is not expressed in striatal interneurons (Supplementary Figs. 1a-c) and the two cre lines are non-overlapping outside of the ventral telencephalon $40,41,44,45$. Thus comparing the phenotypes shared in these two conditional mutants (cKO) allowed us to determine the deficits due to Ebf1 inactivation in dSPN. As expected, Ebf1 was absent from the entire striatal mantle at E17.5 in both cKOs (Fig. 4b-d) and striatal size was slightly reduced, as in $E b f 1^{-1-}$ mice ${ }^{25}$. However, all the generic early SPN markers we examined, including Ctip2 (Fig. 4e-g), Foxp1, Gad1 (not shown), DARPP-32 (Fig. 8j-1) indicated that the striatum was still formed by GABAergic neurons with a SPN-like identity. In order to compare the phenotypes in the two cKOs and gain insights in the molecular programs controlled by Ebf1, we performed RNA-sequencing (RNA-seq) in striatal tissue of control, Islet1 ${ }^{\text {Cre }}$;Ebf $1^{f l-}$ and Dlx5/ 6::Cre;Ebf1 ${ }^{f l}$ - embryos at E17.5 (Fig. $4 \mathrm{~h}-\mathrm{k}$ and Supplementary Data 1 and 2). Importantly, most genes were commonly deregulated in the two cKOs (Figs. $4 \mathrm{~h}-\mathrm{k}$ and Supplementary Data 1 and 2) suggesting that inactivation of Ebf1 in dSPN or all SPN lead to similar phenotypes. To compare the data sets, we examined genes that were previously identified as representative of the core SPN generic identity, versus specific of dSPN or iSPN identity $24,26,28,29,46$. We found that genes associated with a generic SPN identity or with iSPN identity were not significantly altered in both mutants (Fig. 4h, i and Supplementary Data 1 and 2). In contrast, the expression of genes associated with dSPN were either preserved (Drd1, Foxp2, Islet1, Pdyn), or severely deregulated in both mutant models (Fig. 4j, k and Supplementary Data 1 and 2). The preserved expression of Foxp 2 and Islet 1 shows that these transcriptional regulators must act in a partially distinct pathway. Deregulated genes encoded selective transcriptional regulators such as $Z f p 521, M e f 2 c^{27,47,48}$, transmembrane receptors such as PlexinD1, which is involved in synaptogenesis ${ }^{19}$, and intracellular effectors such as $\operatorname{Slc35d3}$, which regulates D1 receptor trafficking ${ }^{49}$. These results were validated by in situ hybridization on selected key genes (Supplementary Fig.4). Finally, Gene Ontology analyses revealed specific alterations of 
a

Control

Islet1 ${ }^{\mathrm{Cre}} ; \mathrm{Ebft}^{\text {fl/ }}$

DIx5/6::Cre;Ebft $1^{f /-}$
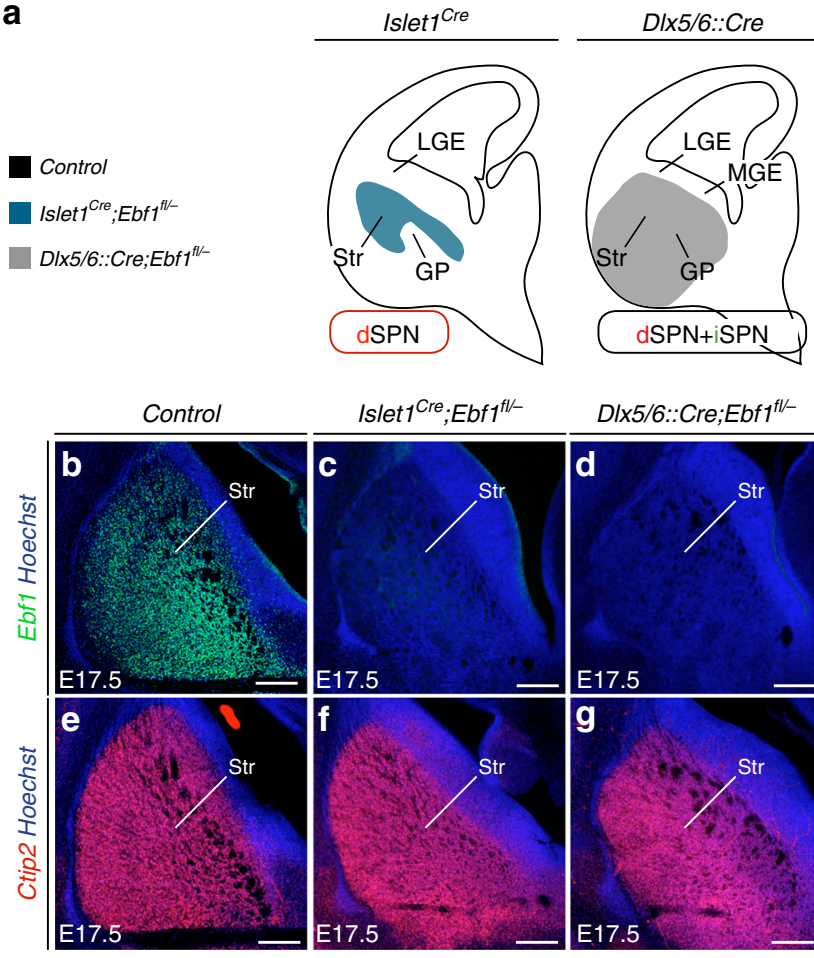

Islet ${ }^{\text {Cre }} ;$ Ebf $^{\text {fl/ }}{ }^{\text {- }}$
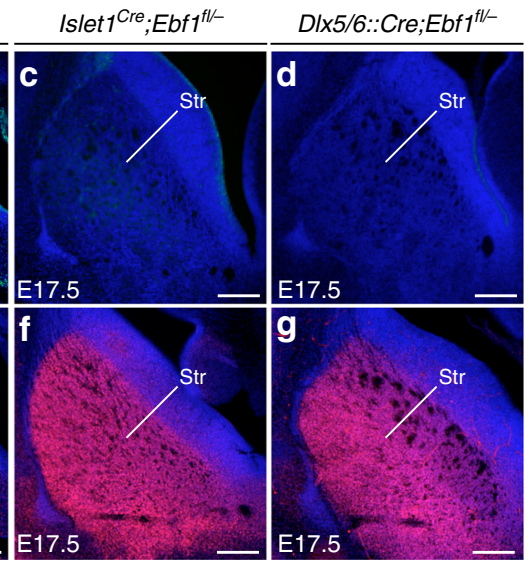

RNA-seq at E17.5
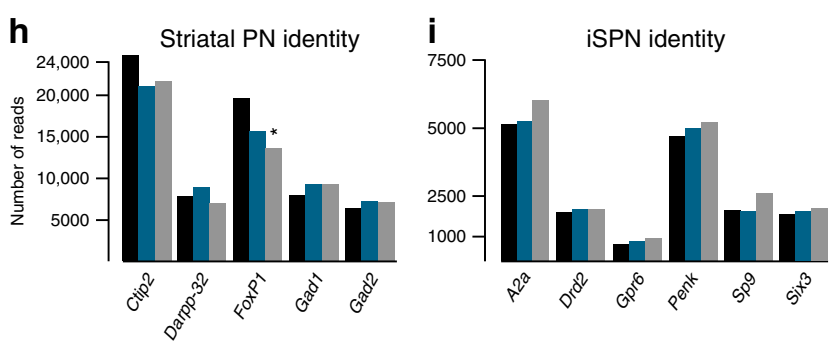

j
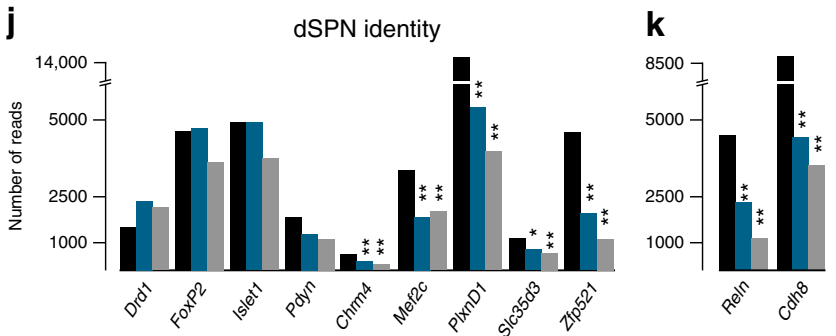

Fig. 4 Two Ebf1 conditional mutants similarly affect dSPN differentiation. a Schematic representations showing that Islet 7 Cre and D/x5/6::Cre lines drive recombination in differentiated dSPN of the mantle and SVZ/mantle SPN, respectively. b-d Immunostaining at E17.5 shows a complete absence of Ebf1 protein in the striatum following conditional deletion with either Islet $7^{\text {Cre }}$ or D/x5/6::Cre lines (at least $n=3$ for each genotype and marker). e-g Expression of the SPN-specific marker Ctip2 is maintained in both Ebf1 CKOs. h-k RNA-sequencing performed at E17.5 on control, Islet7 ${ }^{\mathrm{Cre}}$; $\mathrm{Ebffll}$ and D/x5/6::Cre;Ebffll/ - striatal tissues reveals that genes implicated in SPN (h) or iSPN identity (i) are unaffected whereas subsets of genes associated with dSPN identity (j) as well as the striatum-enriched genes Reln and C $d h 8$ (k) are drastically down-regulated in both cKOs. Importantly, Foxp2 transcription is not significantly affected by Ebf1 cKO at E17.5. Statistical comparison performed with DESeq 1.8 .3 (see Methods section); *indicates adjusted $p$-value < 0.05: ** adjusted $p$-value < 0.001. Scale bar equals 200 $\mu \mathrm{m}$. GP globus pallidus, LGE lateral ganglionic eminence, MGE medial ganglionic eminence, Str striatum factors regulating axon development, cell-cell adhesion properties, and synaptogenesis in both cKOs (Supplementary Figs. 4mo). Taken together, our findings indicate that, while dispensable for the acquisition of a generic SPN transcriptomic profile, Ebf 1 regulates the expression of genes involved in selective aspects of dSPN differentiation.

Ebf1 conditional deletion selectively impairs dSPN differentiation. Since our transcriptomic analysis revealed that $E b f 1$ controls the prenatal expression of major regulators of axon development and synaptogenesis (Fig. 4 and Supplementary Fig. 4), we examined striatonigral and striatopallidal projections in both cKOs (Fig. 5). In P5 controls, DARPP-32 immunostaining labels SPN axons including the ones of dSPN which crossed the globus pallidus (GP) and the entopeduncular nucleus (EP), joined the cerebral peduncle $(\mathrm{CP})$, and reached the substantia nigra (SN) (Fig. 5a). In both cKOs, DARPP- $32+$ projections extended to the EP but only a small fraction of them reached the $\mathrm{SN}$ (Fig. 5a-c), with axonal density in the $\mathrm{CP}$ showing an approximatively $60 \%$ reduction compared to controls (Fig. $5 \mathrm{~d}-\mathrm{g}$ ). In contrast, when we examined striatopallidal projections by measuring the density of Enkephalin signal in the GP we found no major differences between cKOs and controls (Fig. 5h-k). Thus, Ebf 1 inactivation selectively impaired the capacity of dSPN to form the direct pathway.

In contrast to $E b f 1^{-1-25-27}$, cKOs survive up to adult age, allowing us to examine the long-term impact on SPN properties and pathway formation. We observed in cKOs that dtTomatolabeled dSPN were reduced in number and size at P45 in cKO mice (Fig. 6a-g), indicating possible functional impairment. To further analyze the impact of Ebf1 inactivation on dSPN connectivity, we recorded glutamatergic miniature excitatory postsynaptic currents (mEPSCs) in the whole-cell configuration from adult control and $\mathrm{cKO}$ mice. We used either dtTomato or Drd2-EGFP expression as selective SPN subtype markers (Fig. $6 \mathrm{~h}-\mathrm{j}$ ). Moreover, neurons were filled with biocytin during recording for later morphology reconstructions. Consistent with $\mathrm{dtTomato}$ labeling, reconstruction of mutant biocytin-filled neurons showed overall reduced dSPN morphology, in contrast to iSPN (Fig. 6k-m). In addition, in both cKOs, dSPN mEPSC frequency was severely reduced, with most cells presenting no detectable spontaneous synaptic activity. In contrast, iSPN did not show significant differences from controls (Fig. $6 \mathrm{~g}-\mathrm{l}$ and Supplementary Figs. 5a-f). We further examined the functional impact on direct and indirect pathways using a pharmacological approach in vivo, by testing motor responses to injections of either D1 or D2 receptor agonists ${ }^{24}$. In baseline conditions, we found that neither cKOs showed major locomotion deficits, Dlx5/ $6:: C r e ; E b f f^{f /-}$ mutants exhibiting only a mild hyperactivity phenotype (Supplementary Fig. 5g). Such phenotype, which is likely due to extra-striatal defects, precludes a full interpretation of pharmacological challenges (Supplementary Fig. 5). We thus focused on Islet $1^{\mathrm{Cre}}$; Ebf $\mathrm{I}^{\mathrm{fl} / \mathrm{-}}$ mutants and first activated the direct pathway by injection of the D1 receptor agonist SKF82958 (Fig. 6n). In contrast to control mice, D1 receptor agonist injections in Ebf1 cKOs did not lead to significant locomotion increases, indicating important functional disruptions of the direct pathway in line with the anatomical defects observed (Fig. 5a-f). Conversely, activation of the indirect pathway through injections of the D2 receptor agonist quinpirole (Fig. 6o) caused strong, statistically similar decreases in the mobility in both control and cKOs, confirming that direct pathway functions are selectively affected by Ebf1 inactivation. Notably, we found that the indirect pathway is also functionally preserved in Dlx5/6::Cre; Ebfil ${ }^{f /-}$ mutants (Supplementary Fig. 5h). 


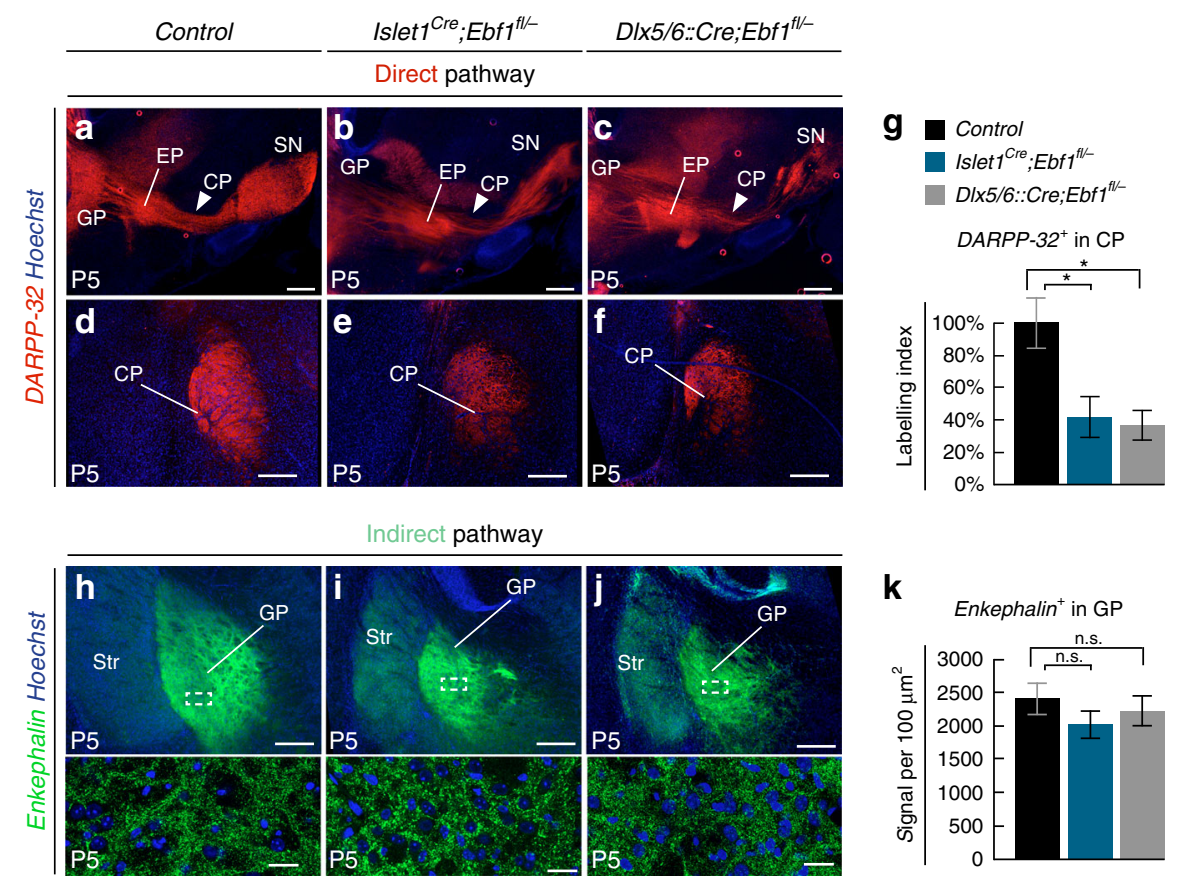

Fig. 5 Ebf1 conditional inactivation affects direct pathway formation. a-c DARPP-32 staining of striatal axons on P5 sagittal sections reveal that, instead of reaching their target in controls $(n=3)(\mathbf{a})$, striatofugal axons show limited extension passed the entopeduncular nucleus (EP) in both $/$ slet 7 Cre; $E b f f l /-$ ( $n=$ 4) (b) and D/x5/6::Cre;Ebffll- $(n=4)$ (c) mutants (solid arrowheads). d-g DARPP-32 staining in coronal sections showing reduced axonal projections in the cerebral peduncle (CP) of Ebf1 cKOs, quantified in ( $\mathbf{g}$ ). Labeling Index is a function of signal area and mean intensity ( $p=0.028$ for Islet 7 Cre $E b f f l /-$ and $p=0.023$ for Dlx5/6::Cre;Ebffl/-). h-k Indirect pathway terminals in GP, labeled by Enkephalin immunostaining, show similar densities in controls and both

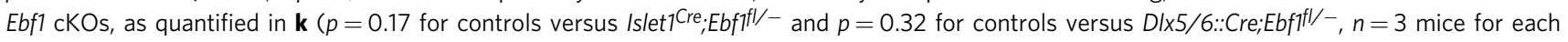
condition). Results are presented as mean values \pm s.e.m. Two-tailed non-parametric Mann-Whitney $U$ test was used for statistical comparison. * indicates p-value $<0.05$. Scale bars equal $400 \mu \mathrm{m}$ (a-c), $200 \mu \mathrm{m}$ (d-j), and $25 \mu \mathrm{m}$ (bottom insets). CP cerebral peduncle, EP entopeduncular nucleus, GP globus pallidus, SN substantia nigra, Str striatum

Our results reveal that development and functioning of the direct pathway is selectively impaired in adult Ebf1 cKOs, while cardinal properties of iSPN, including circuit integration and electrophysiological properties, are preserved. Thus, iSPN differentiation and maturation are largely independent of dSPN.

Ebf1 deletion affects iSPN migration and intermix in the striatal matrix. Next, we examined whether, in addition to its role in dSPN differentiation, Ebf1 could play a role in SPN intermix and striatal mosaic formation. As is the case for other dSPN markers such as Islet 1 and Drd1 (Fig. 4 and Supplementary Fig. 4), the transcriptional levels of FoxP2 were preserved in both early Ebf1 cKOs (Fig. 4 and Supplementary Fig. 4). Therefore, we used its expression to analyze the distribution of dSPN in the embryonic striatum. We consistently observed non-overlapping populations of $\mathrm{Ctip}^{+} \mathrm{FoxP}^{+} \mathrm{dSPN}$ and Ctip2 ${ }^{+}$Drd2-EGFP $^{+}$ iSPN in mutant striata (Fig. 7). However, we found that dSPN and iSPN distribution was strikingly different in the striatum of controls and of the two cKOs at E17.5 (Fig. 7). Indeed, on one side the density of dSPN in both cKOs was reduced in the dorsal striatum and increased in the lateral region in comparison to controls (Fig. 7a-k). Conversely, the density of Drd2-EGFP + iSPN was enhanced dorsally and reduced laterally (Fig. 7l-u). Thus, although the overall respective proportions of dSPN and iSPN were preserved, Ebf1 inactivation perturbed the distribution of the two SPN populations.

The dorsal accumulation of iSPN near progenitor zones raised the possibility that their migration might be indirectly impaired in Ebf1 cKO. To test this hypothesis, we first performed timed EdU injections to follow SPN progression into the striatal mantle of Ebf1 mutants, using Drd2-EGFP and either Foxp2 or
dtTomato as respective markers of iSPN and dSPN (Supplementary Fig. 6). We observed that EdU-stained iSPN abnormally accumulated in the dorsolateral part of the striatum in cKOs (Supplementary Fig. 6), suggesting that Ebf1 non-cellautonomously regulates their migration. To directly monitor iSPN migration, we performed two-photon time-lapse imaging in E15.5 Islet $1^{C r e} ; E b f 1_{1}{ }^{f l-} ;$; Drd2-EGFP and Dlx5/6::Cre;Ebf $1^{f /-} ;$;rd2EGFP slices. We found that in both genetic backgrounds, mutant iSPN still harbored pattern of multidirectional migration as in controls (Fig. 7v and Movies 2-4). However, analyses over several time-lapse movies showed that iSPN average speed was significantly decreased (Fig. $7 \mathrm{w}$ ), thereby revealing that Ebf1 non-cell-autonomously controls the migration efficiency of iSPN. Thus Ebf1 inactivation in dSPN impairs iSPN migration and their process of intermixing. Since Ebf1 and Islet1 both control dSPN differentiation, we investigated the specificity of such intermixing defect by comparing the distribution of D2 receptor (Drd2) transcripts in Ebf1 and Islet1 cKOs (Supplementary Fig. 7). In contrast to $E b f 1$ cKO, we found that iSPN distribution was not drastically altered in Islet $1 \mathrm{cKO}$ (Supplementary Fig. 7). Therefore, iSPN intermixing specifically relies on Ebf1 expression in dSPN, indicating a novel and specific role for this transcription factor in the acquisition of dSPN properties.

In addition to changes in dorso-ventral distribution, we observed that iSPN presented a higher degree of segregation, especially in the lateral region (Fig. 7l-t). This observation raised questions about possible disruptions of the striosomes/matrix organization. Using early striosome markers such as DARPP- $32^{50}$ (Supplementary Fig. 1), we found that the two compartments could be unambiguously identified in both cKOs (Fig. 8a-c). However, we observed that the matrix contained Enkephalinhigh 

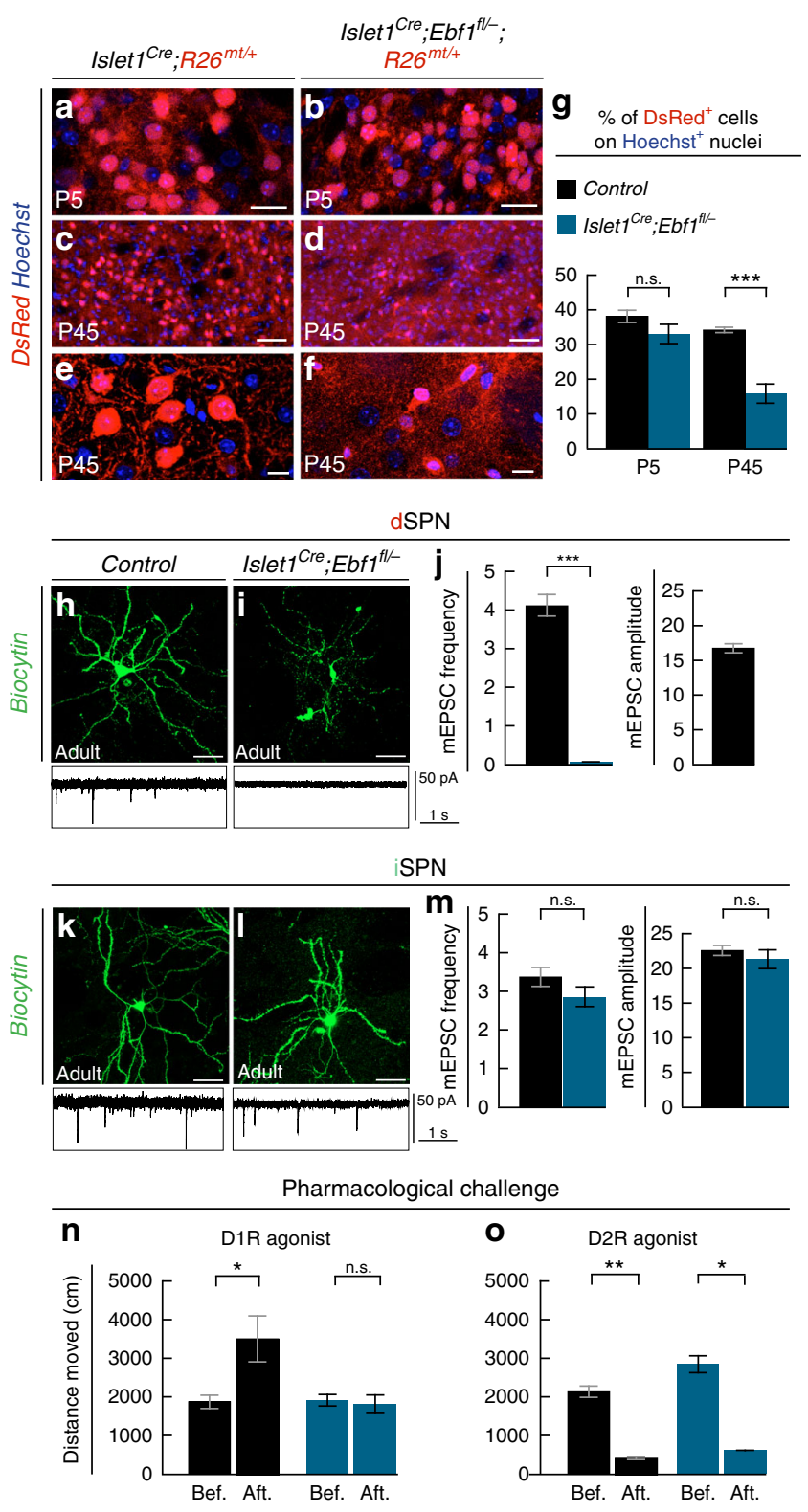

domains (Fig. 8d-f) which segregated from cellular islands containing matrix dSPN (Supplementary Fig. 8) in both cKOs, suggesting an intermixing deficit between iSPN and dSPN. To further test this possibility, we examined Drd2-EGFP controls and cKOs at E17.5, when compartment segregation is largely engaged $14,15,30-33,38$ and proto-striosomes can be identified by the expression of Substance $P$ (not shown) and DARPP-32 (Fig. 8j-1), a maturation marker, which is turned on in the matrix only at later stages. We observed that the deficit in iSPN and $\mathrm{dSPN}$ intermixing was more pronounced in the matrix (Fig. 8k-1), consistently with previous reports showing that Ebf1 inactivation more specifically affects matrix $\mathrm{dSPN}^{27}$. This specificity was further highlighted by the finding that dopaminergic innervation is selectively impaired in matrix dSPN-rich territories (Supplementary Fig. 9). Collectively, our findings reveal that the intermixing of iSPN and dSPN in the matrix specifically relies on Ebfl expression in dSPN (Fig. 8m-n). This major step in the emergence of a balanced striatum parallels the formation of compartments, thereby revealing that multiple migratory processes regulate the assembly of the striatal mosaic (Supplementary Fig. 10).
Fig. 6 Ebf1 cKO have specific impairments in dSPN functioning. a-g

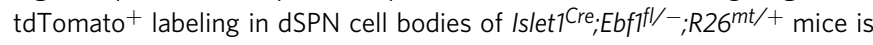
relatively preserved at early postnatal stages $(\mathbf{a}, \mathbf{b})$ but severely altered at later stages (c-f), as quantified in $\mathbf{g}$; at P5, the percentage of DsRed + cells on the Hoechst nuclei is $38 \pm 2 \%$ in Control striata, $33 \pm 3 \%$ in Islet7 Cre; Ebffll/- $(p=0.16)$. At P45, Control: $34 \pm 1 \%$, Islet 7 Cre, Ebff fl/ $-16 \pm 3 \%$ ( $p=$ $0.002) . n=3$ mice for each condition. Results are presented as mean values \pm s.e.m. $\mathbf{h}$-j Patch-clamp recordings of dSPN in acute slices obtained

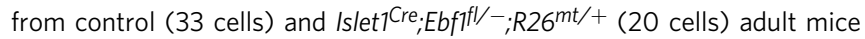
highlight a drastic decrease of $m E P S C$ frequency in mutants (sample tracks in $\mathbf{g}$-h, quantification of $\mathrm{mEPSC}$ in $\mathbf{i}) \mathbf{k}$ - $\mathbf{m}$ Conversely, iSPN recorded in controls (27 cells) and Islet 7 Cre; Ebff $7^{f / /} ; R_{2} 6^{\mathrm{mt} /+}$ (14 cells) slices show no relevant differences in $\mathrm{mEPSC}$ amplitude and frequency. $\mathrm{mEPSC}$ amplitudes could not be quantified in mutant dSPN because of the extremely low number of events recorded. Results are presented as mean values \pm s.e.m. n, o Adult Islet7 ${ }^{C r e}$;Ebff $f /$ - mice show no motor response to D1R agonist SKF38393 injections (n), whereas the D2R agonist quinpirole induces depression of locomotion similarly to control mice (0). This is shown by comparing the total distance traveled in $10 \mathrm{~min}$ in the open field before (Bef.) and $40 \mathrm{~min}$ after (Aft.) drug injections $\left(n_{\text {control }}=8\right.$ and $\left.n_{\mathrm{cKO}}=7\right)$. Results are presented as mean \pm standard deviation. Two-tailed nonparametric Mann-Whitney $U$ test was used for statistical comparison. * indicates $p$-value $<0.05,{ }^{\star \star \star} p$-value $<0.0001$. Scale bars equal $20 \mu \mathrm{m}(\mathbf{a}, \mathbf{b}$, e, f), $100 \mu \mathrm{m}$ (c, d), and $40 \mu \mathrm{m}$ (g-k), respectively

\section{Discussion}

Striatal functioning depends on the balanced activation of intermixed direct and indirect pathway neurons ${ }^{51}$. It is thus essential to understand how the two subtypes are specified and how they assemble into a mosaic striatal architecture. Here, we show that while dSPN and iSPN differentiation is largely independent, their intermixing relies on a developmental crosstalk, which is crucial to organize striatal architecture and is largely independent from striosome and matrix compartment formation.

While both SPN derive from the $\mathrm{LGE}^{13,16,17,31}$, producing fully differentiated $\mathrm{dSPN}$ or iSPN in vitro ${ }^{52}$ has remained a challenge. While there was increasing evidence that distinct transcriptional programs underlie the specification of both subtypes $16,24,28,29$, in vitro studies raised questions regarding the mechanisms controlling SPN differentiation. Our results confirm that dSPN and iSPN possess distinct genetic fingerprints from early embryonic stages, reinforcing the idea that the two cell types are already specified when they enter the striatal mantle.

Furthermore, iSPN progressively invade dSPN-rich areas through dynamic multidirectional migration. Intriguingly, iSPN are initially detected in the lateralmost part of the striatum, consistently with the observation that transcription factors regulating iSPN development, Six 3 and Sp9, are located in the dorsal part of the $\mathrm{LGE}^{16}$. Tangential migration is a well-known mechanism for increasing neuronal diversity in different brain structures. In the telencephalon, tangential migration has mostly been associated with interneuron behavior ${ }^{53}$, but has also been involved in the positioning of other LGE-derived projection neurons, including arkypallidal neurons of the globus pallidus $^{54,55}$, corridor neurons ${ }^{56,57}$ and dSPN-like neurons of the central $^{58}$ and extended amygdala ${ }^{59}$. Further investigation will be required to determine the cellular and molecular mechanisms governing the migration and interaction of different LGEgenerated populations. Nevertheless, tangential migration of projection neurons is emerging as a shared mechanism to build and increase cellular diversity in basal ganglia nuclei, in contrast to the laminated cerebral cortex where projection neurons migrate radially from the underlying proliferative neuroepithelium ${ }^{60}$. 


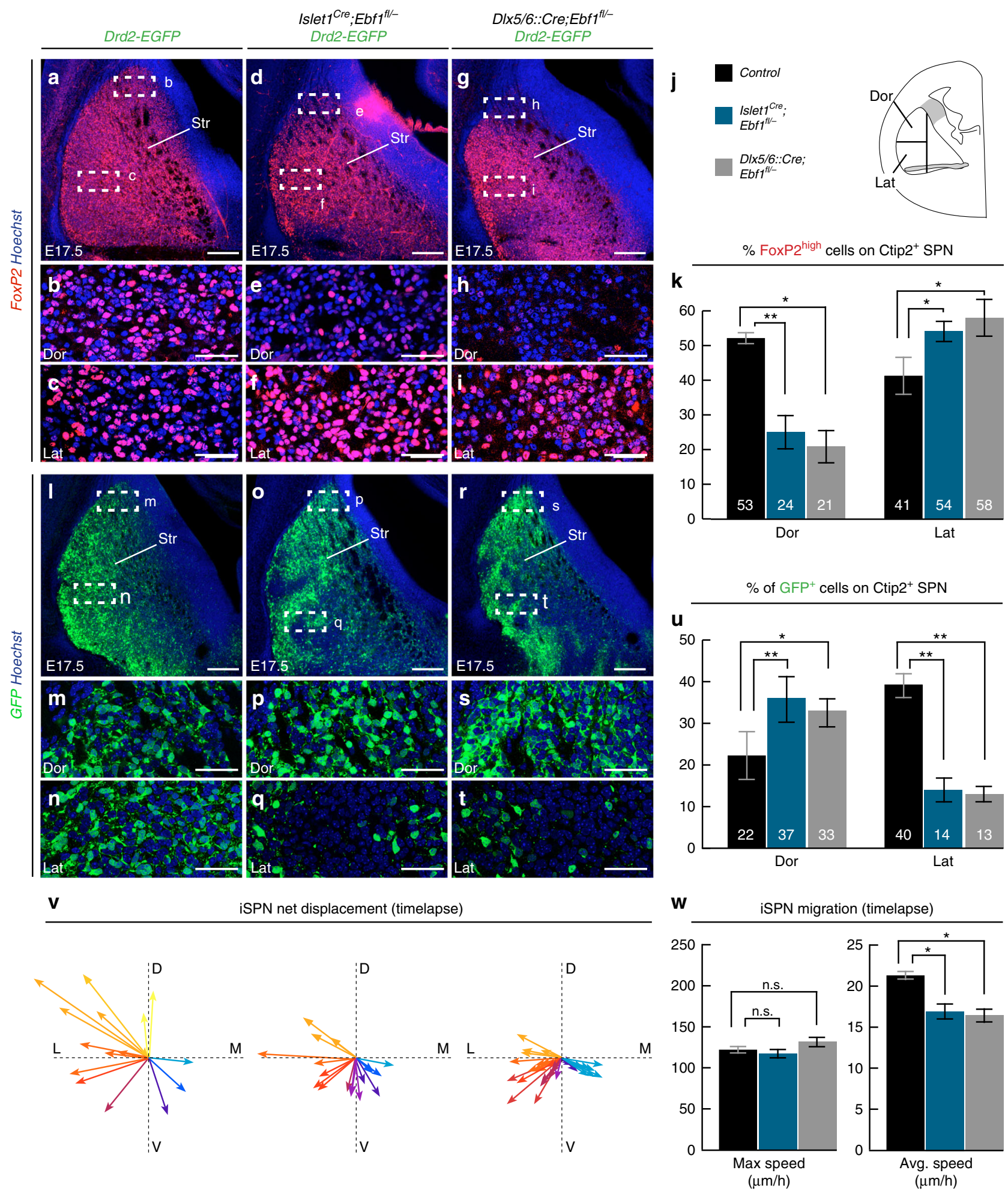

Regarding the transcriptional cascades governing SPN identity, our work indicates that Ebf1 plays a major role in dSPN differentiation, independently of the other known regulators Islet1 and Foxp2. Ebf1 is a member of the COE family that plays a key role in the specification of $B$ cells and the differentiation of neuronal subtypes $^{52,61-63}$. Ebf1 acts both as a direct regulator of downstream target genes ${ }^{61-63}$ and as a chromatin remodeler, poising or regulating the accessibility of enhancers ${ }^{64,65}$. Ebfl is expressed in the developing striatum ${ }^{25}$ and analyses of Ebfl full knockout mice showed normal proliferation in the LGE but defective survival of matrix dSPN and disorganized projections to the $\mathrm{SN}^{26,27}$. By combining analysis of two distinct cKOs, we first showed that Ebf1 is dispensable for the fate choice between dSPN and iSPN identity and the core aspects of differentiation into GABAergic SPN neurons. Indeed, Ebf1 inactivation leads to an abnormal differentiation of dSPN-like neurons that nonetheless retain their GABAergic identity and expression of DARPP-32, Ctip2, FoxP1, Drd1, Islet1, and FoxP2. Notably, deregulated genes included 
Fig. 7 Ebf1 cKO affects iSPN migration and intermix with dSPN. a-j Distribution of FoxP2 ${ }^{\text {high }}$; Ctip2 + dSPN is altered in Ebf1 cKOs at E17.5, with lower density in the dorsal (Dor) and higher in the lateral (Lat) part of the striatum (Str); the two regions are defined in ( $\mathbf{j}$ ) ( $n=3$ for each condition). $\mathbf{k}$ In control Dor Str, $53 \pm 3.5 \%$ of all Ctip2 ${ }^{+}$SPN are Foxp2 ${ }^{+}, 24 \pm 6 \%(p=0.0079)$ in Islet 7 Cre; Ebff fl/ - and $21 \pm 8 \%$. $(p=0.03)$ in D/x5/6::Cre; Ebffl/ - . In control Lat Str, Foxp2 + cells form $41 \pm 11 \%$ of Ctip2 + SPN, raising to $54 \pm 3 \%$ in Islet7Cre;Ebffll/- $(p=0.01)$ and to $58 \pm 11 \%$ in D/x5/6::Cre; Ebffll/- $(p=0.03)$. I-t Distribution of Drd2-EGFP+;Ctip2 ${ }^{+}$iSPN is altered by Ebf1 cKO, with higher density in Dor Str and lower in Lat Str. u Quantification of iSPN distribution. In control Dor Str, $22 \pm 12 \%$ of Ctip2 ${ }^{+}$SPN are GFP+. This percentage increases to $37 \pm 11 \%$ in Islet 7 Cre; Ebffl/ $-;$ Drd2-EGFP ( $\left.p=0.007\right)$ and to $33 \pm 7 \%$ in D/x5/ $6:: C r e ; E b f f l / /-; \operatorname{Drd} 2-\operatorname{EGFP}(p=0.03)$. Conversely, in the Lat Str, the percentage of of double-positive GFP ${ }^{+} \mathrm{Ctip} 2+$ iSPN equals $40 \pm 6 \%$ in controls, $14 \pm 6 \%$

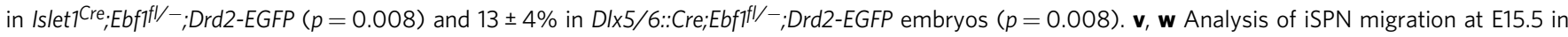

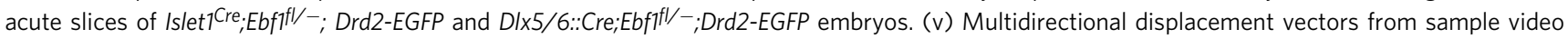

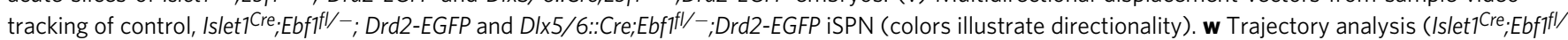
-; Drd2-EGFP: 58 cells tracked, $\mathrm{n}=3$ independent experiments; DIx5/6::Cre;Ebf1 fl/-;Drd2-EGFP 29 cells tracked, $\mathrm{n}=2$ independent experiments) shows

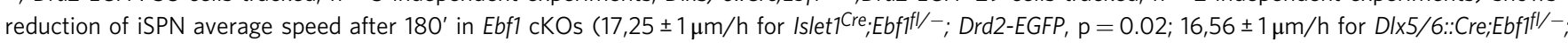

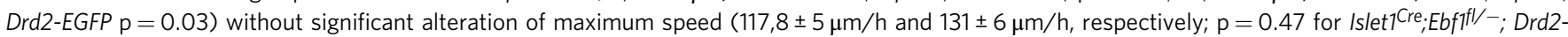
EGFP and $\mathrm{p}=0.55$ for D/x5/6::Cre;Ebffll/-;Drd2-EGFP when compared to control iSPN). Results are presented as mean \pm standard deviation ( $\mathrm{k}$, $\mathrm{u}$ ) or s.e.m (w). Two-tailed non-parametric Mann-Whitney $U$ test was used for statistical comparison. ${ }^{\star}$ indicates $p$-value $<0.05,{ }^{\star \star} p$-value $<0.01$. Scale bars equal $200 \mu \mathrm{m}(\mathrm{a}, \mathrm{d}, \mathrm{g}, \mathrm{l}, \mathrm{o}, \mathrm{r}), 50 \mu \mathrm{m}(\mathrm{b}, \mathrm{c}, \mathrm{e}, \mathrm{f}, \mathrm{h}, \mathrm{l}, \mathrm{m}, \mathrm{n}, \mathrm{p}, \mathrm{q}, \mathrm{s}, \mathrm{t})$. Dor, Dorsal; Lat, lateral; Str, striatum

Zfp521 and Mef2c, which are directly bound by Ebf1 in pro-B cells and thus likely direct targets ${ }^{61,62}$. Second, Ebf1 is not expressed in the ventral striatum and its inactivation selectively perturbs the matrix compartment of the dorsal striatum, potentially revealing a subdivision of dSPN neurons. Third, Ebf1 regulates expression of genes controlling axon and synaptogenesis, thereby highlighting a major role in circuit integration. Importantly, Ebf1 and Islet 1 both regulate direct pathway formation and the expression of PlexinD 19,24,25,28, suggesting that the two genes might cooperatively regulate specific features of $\mathrm{dSPN}$ neurons.

Finally, we found that Ebf1 non-cell-autonomously regulates iSPN intermixing with dSPN in the matrix compartment. In Ebf1 cKOs, iSPN are correctly specified and functional, but their progression and intermixing with dSPN is impaired. This intermixing deficit occurs independently of the formation of striosomes/matrix and is more pronounced in the matrix compartment. Remarkably, this phenotype is absent in Islet 1 $\mathrm{cKO}$, indicating a novel and specific role for Ebf1 in the building of striatal functional organization. The underlying mechanisms remain to be deciphered and could include the production of membrane-bound or secreted factors acting on iSPN. Our study also reveals that the intermix of dSPN/iSPN and the formation of striosomes/matrix compartments constitute parallel processes. Indeed, it has been shown that early-born and late-born SPN interact in late embryogenesis to respectively form the striosomes and the matrix via migration and ephrinA/EphA4-dependant cell-sorting 14,15,17,31-33,38. In Ebf1 cKOs, striosomes do form but the intermix of matrix neurons is defective. Our study thus reveals that distinct steps of migration and reorganization control the emergence of two compartments comprising intermixed dSPN/ iSPN (Supplementary Fig. 10), an architecture which is essential for striatal functioning.

Taken together, our work shows that while iSPN and dSPN specification is largely independent, the assembly of striatal mosaic emerges from a dSPN-dependent tangential migration of iSPN. This study establishes a novel framework for the formation of the striatum, a major structure associated with developmental disorders, and provides key insights on how migration controls the wiring of neural circuits.

\section{Methods}

Mouse lines. For fate mapping studies, Islet $1^{C r e /}+$ animals $^{43}$ were crossed with either R26 $6^{m t / m t}$ or R26 $6^{m t / m t}$;Drd2-EGFP mice ${ }^{34}$. To obtain Islet $1^{C r e /+} ; E b f 1^{f l-}$, Islet $1^{\mathrm{Cre} /+} ; \mathrm{Ebf1}^{\mathrm{fl} /-} ; \mathrm{R}^{\mathrm{mt} /+}$, and Islet $1^{\mathrm{Cre} /+}:$ Ebf1 ${ }^{\mathrm{fl} /-}$;Drd2-EGFP conditional mutants, we initially crossed Islet $1^{\text {Cre/+ }}$ mice with Ebf $1^{+/-66}$ to generate Islet $1^{\text {Cre/+}}$; $E b f 1^{f l-}$ animals. These were in turn backcrossed with either Ebf1 fl/fl 62,67, Ebf1 fl/fl; $R 26^{m t / m t}$, or Ebf1 ${ }^{f l / f l}$;Drd2-EGFP mice, respectively. Similarly, Dlx5/6::Cre;Ebf $1^{f l /-}$ and Dlx5/6::Cre;Ebf1 ${ }^{f l /}$;Drd2-EGFP mice were obtained by crossing Dlx5/6::Cre $\mathrm{C}^{44}$ mice with $E b f 1^{+/-}$to generate $D l \times 5 / 6:: C r e ; E b f^{+/-}$animals, which were in turn backcrossed with Ebf1 $1^{\text {fl/fl }}$ or Ebf1 ${ }^{f l / f}$;Drd2-EGFP mice. All transgenic lines were maintained on a C57/Bl6 background, with the exception of Islet $1^{\mathrm{Cre} /+}$ and Drd2EGFP lines that remained on a B6D2F1/J genetic background. Heterozygous embryos did not show any phenotype and were used as controls. Nkx2.1 ${ }^{\text {Cre } /+}$ mice provided by S. Anderson's laboratory ${ }^{68}$. $12-\mu \mathrm{m}$-thick cryosections from $\mathrm{Dlx1} \mathrm{Cre}^{\mathrm{C} /+}$; Islet $1^{f l f l}$ embryos provided by K. Campbell's laboratory ${ }^{24}$. The day of vaginal plug was considered as embryonic day (E) 0.5 and day of birth as postnatal day (P) 0 . Animals were bred under French and EU regulations, following recommendations of the Charles Darwin ethics committee.

In situ hybridization and immunohistochemistry. For in situ hybridization, brains were fixed overnight in $4 \%$ paraformaldehyde in PBS (PFA) at $4{ }^{\circ} \mathrm{C} .80$ to $100-\mu \mathrm{m}$-thick free-floating vibratome sections (Leica S1000) were hybridized as described; ${ }^{67} \mathrm{Dl} \times 1^{\mathrm{Cre} /+} ;$ Islet $1^{f l / f l}$ and respective control cryosections were hybridized as described ${ }^{69}$. For immunohistochemistry, mice were perfused with $4 \%$ PFA. Brains were dissected and post fixed overnight at $4{ }^{\circ} \mathrm{C}$. Immunohistochemistry was performed on $60-\mu \mathrm{m}$-thick free-floating vibratome sections. Slices were incubated $1 \mathrm{~h}$ at room temperature (RT) in a blocking solution containing $0,25 \%$ Triton X100 (Sigma), 0,02\% Gelatine in PBS, and incubated in the same blocking solution with primary antibodies overnight at $4{ }^{\circ} \mathrm{C}$. Primary antibodies were used at the following concentrations: rat anti-CTIP2 1/500 (Abcam), mouse anti-DARPP-32 1/ 100 (Santa Cruz), rabbit anti-DARPP-32 1/1000 (Millipore), rabbit anti-DsRed 1/ 500 (Living colors), rabbit anti-Enkephalin 1/500 (Millipore), rabbit anti-Ebf1 1/ 250 (Abcam), rabbit anti-FoxP1 1/200 (Abcam), goat anti-FoxP2 1/200 (Santa Cruz), chicken anti-GFP 1/1000 (Aves), rabbit anti-Slc35d3 1/250 (Novusbio), rat anti-Substance P 1/400 (Millipore), rabbit anti-Tyrosine Hydroxylase 1/1000 (Abcam), guinea pig anti-vGlut1 1/10000 (Millipore), and guinea pig anti-vGlut2 $1 / 10000$ (Millipore). Sections were rinsed several times in PBS and incubated from $2 \mathrm{~h}$ to overnight at $4{ }^{\circ} \mathrm{C}$ with the appropriate fluorescent secondary antibodies: A488-conjugated donkey anti-rabbit, anti-rat or anti-chicken, Cy3-conjugated donkey anti-rat, anti-goat, anti-mouse, Cy5-conjugated donkey anti-rabbit or antirat, DyLight488-conjugated donkey anti-guinea pig (1/400, all antibodies from Jackson ImmunoResearch). Hoechst (Sigma) was used for fluorescent nuclear counterstaining and sections were mounted in Mowiol or Vectashield (Vector).

Birthdating. Pregnant dams were injected intraperitoneally at the appropriate gestation day with a solution containing 5-Ethynyl-2'-deoxyuridine (EdU, Thermo Fisher). $60-100-\mu \mathrm{m}$-thick free-floating vibratome sections were processed following manufacturer instructions (Click-iT EdU Alexa Fluor 488 Imaging kit, Life Technologies) for $30 \mathrm{~min}$ at RT. Sections were rinsed three times in 3\% BSA and then in PBS. Hoechst staining was performed for $30 \mathrm{~min}$ at RT before pursuing the immunohistochemistry protocol as described above.

Image acquisition, analysis, and quantification. Images were acquired on a fluorescence microscope (Leica MZ16 F), a fluorescent microscope (Leica DM5000 B) or a confocal microscope (Leica TCS SP5). Images were then processed with ImageJ and Adobe Photoshop software. For cell density and colocalization analysis, three different rostro-caudal striatal levels of the striatum were initially defined, using the anterior commissure as an anatomical landmark. Single-plane confocal images were taken at each level in three different animals for each condition. For cell distribution analysis in Fig. 7, a high-definition tilescan of the whole striatum at medial level was imaged on a single confocal plane in at least three different animals for each condition. The tilescans were later subdivided in lateral and dorsal regions (Fig. 7d) for quantification. Cell counting was performed semiautomatically using built-in functions in ImageJ. For each experiment, sample 


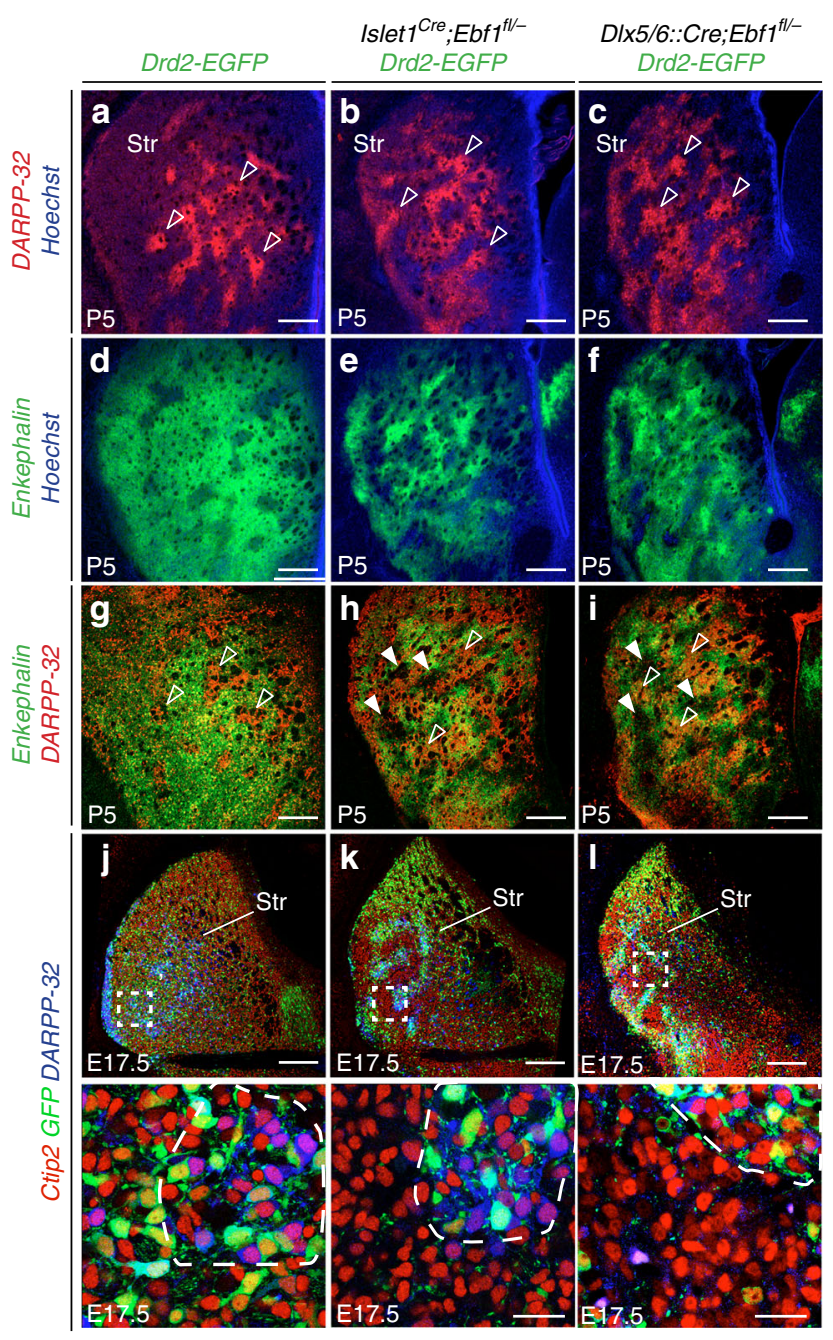

m

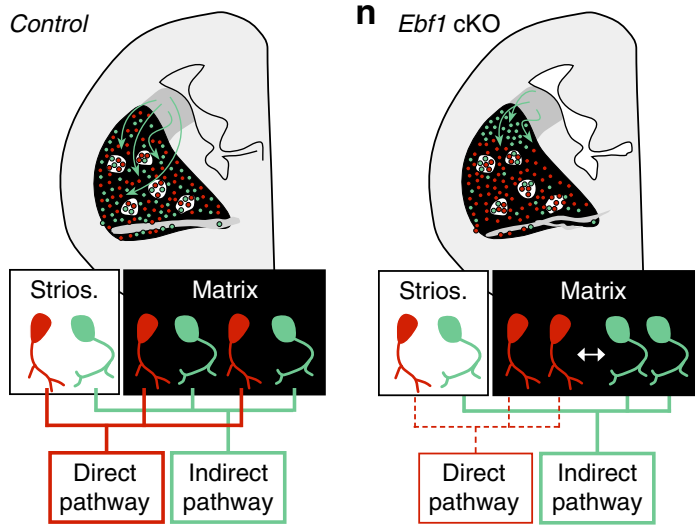

images were manually counted to double-check the quality of semi-automated counting.

Time-lapse imaging and analysis. E13.5 and E15.5 Drd2-EGFP and Dlx5/6::Cre; $E b f 1^{f l-}$;Drd2-EGFP embryos were dissected and kept in an ice-cold solution consisting of L-15 Medium (SIGMA-Aldrich) supplemented with 3\% Glucose. Brains were included in 3,5\% low-melting agarose (Promega) in L-15 Glucose ${ }^{+}$ solution and $350-\mu \mathrm{m}$-thick coronal slices were cut on a vibratome. Slices were imaged using a multi-photon microscope (DIMM, IBENS Imaging Platform) over a period of up to 240 ', while being constantly perfused with a L-15 Glucose ${ }^{+}$ solution at $37^{\circ} \mathrm{C}$ and bubbled with $\mathrm{O}_{2} / \mathrm{CO}_{2}(95 \% / 5 \%) .100-\mu \mathrm{m}$-thick z-stacks were imaged every 6 ' for each single frame. For time-lapse movie analysis, eventual drift in the three dimensions was first corrected using IMARIS software (Bitplane, Oxford); subsequently, $\mathrm{z}$-stacks were flattened on a two-dimensional plane. The
Fig. 8 Defective intermixing is observed in the matrix compartment. a-c DARPP-32 staining in P5 striatal coronal sections reveals that striosomes are still recognizable in Islet7Cre;Ebffll/- and D/x5/6::Cre;Ebffl/- (empty arrowheads) ( $n=3$ for each genotypes and markers). d-i Conversely, in Ebf1 cKOs the matrix compartment is parceled in Enkephalin-rich and Enkephalin-poor (full arrowheads) areas. j-I Intermixing anomalies are prominent in the matrix compartment of both CKOs. In the lateral striatum, iSPN are intermixed both within and outside DARPP-32 + striosomes in controls ( $\mathbf{j}$ and high magnification), whereas iSPN are intermixed in striosomes (delineated by dotted lines) but not in the matrix of Ebf1 cKOs (high magnifications). $\mathbf{m}, \mathbf{n}$ Schematic representation of the functions of Ebf1 in dSPN differentiation and in the non-cell autonomous regulation of iSPN migration. $n=3$ for each genotype. Scale bars equal $200 \mu \mathrm{m}(\mathbf{a}-\mathbf{I})$ and $30 \mu \mathrm{m}$ (bottom panels, high magnification). Str striatum

high number of cells present in each single movie rendered automated analysis of cell displacement unadvisable. Therefore, single cell displacement was measured manually frame-by-frame using the "Manual Tracking" plugin in ImageJ. Cells included in the analysis responded to two criteria: being readily identifiable from the first to the last frame of the movie and showing a total net displacement of more than $50 \mu \mathrm{m}$. In order to examine directionality and migratory pattern of iSPN migration, stationary (total net displacement less than $50 \mu \mathrm{m}$ ) cells were not included in the analysis.

RNA-sequencing. E17.5 Dlx5/6::Cre;Ebf1 ${ }^{f l-}$, Islet $1^{\mathrm{Cre} /+} ; \mathrm{Ebf1} \mathrm{f}^{\mathrm{fl} /-}$ and control embryos were dissected in RNAse-free conditions on ice. Brains were conserved in RNAlater stabilization reagent (Qiagen) solution. Following genotype identification via PCR, messenger RNA was obtained from $\mathrm{n}=3$ brains from each condition using RNeasy mini kit (Qiagen). Library preparation and Illumina sequencing were performed at the Ecole Normale Supérieure Genomic Platform (Paris, France). Messenger (polyA + ) RNAs were purified from 400 ng of total RNA using oligo (dT). Libraries were prepared using the strand specific RNA-Seq library preparation TruSeq Stranded mRNA kit (Illumina). Libraries were multiplexed by 9 on run Nextseq 500. A 75 bp read sequencing was performed on a Nextseq 500 device (Illumina). A mean of $37.5 \pm 8.95$ million passing Illumina quality filter reads was obtained for each of the 9 samples. Analyses were performed using the Eoulsan pipeline, including read filtering, mapping, alignment filtering, read quantification, normalization and differential analysis. Before mapping, poly $\mathrm{N}$ read tails were trimmed, reads $\leq 40$ bases were removed, and reads with quality mean $\leq 30$ were discarded. Reads were then aligned against the Mus musculus genome from Ensembl version 81 using STAR (version 2.4.0k). Alignments from reads matching more than once on the reference genome were removed using Java version of samtools. To compute gene expression, Mus musculus GFF3 genome annotation version 81 from Ensembl database was used. All overlapping regions between alignments and referenced genes were counted using HTSeq-count 0.5.3. Sample counts were normalized using DESeq 1.8.3. Statistical treatments and differential analyses were also performed using DESeq $1.8 .3^{70}$.

Slice preparation and electrophysiological recordings. In vitro electrophysiological recordings were performed in coronal slices from the dorsal striatum of control animals (either Islet $1^{\text {Cre } /+} ; R 26^{m t /+}$ or Drd2-EGFP mice), Dlx5/6::Cre; $E b f 1^{f l-} ;$ Drd2-EGFP, and Islet $1^{C r e /+} ; E b f 1^{f l /-} ; R 26^{m t /+}$ mice. Mice were anesthetized with isofluorane before decapitation. After isolation, the portion of the brain containing the striatum was placed in bicarbonate-buffered saline (BBS) at $2-5{ }^{\circ} \mathrm{C}$ for a few minutes. Slices $(300 \mu \mathrm{m})$ were then cut using a $7000 \mathrm{smz}-2$ vibratome (Campden Instruments Ltd.). The slicing procedure was performed in an ice-cold solution containing (in $\mathrm{mM}$ ): 130 potassium gluconate, $15 \mathrm{KCl}, 0.05 \mathrm{EGTA}, 20$ Hepes, 25 glucose, $1 \mathrm{CaCl}_{2}$, and $6 \mathrm{MgCl}_{2}$. Slices were then briefly transferred to a solution containing (in mM): $225 \mathrm{D}$-mannitol, $2.5 \mathrm{KCl}, 1.25 \mathrm{NaH}_{2} \mathrm{PO}_{4}, 25$ $\mathrm{NaHCO}_{3}, 25$ glucose, $1 \mathrm{CaCl}_{2}$, and $6 \mathrm{MgCl}_{2}$, and finally stored for the rest of the experimental day at $33^{\circ} \mathrm{C}$ in oxygenated BBS, containing: $115 \mathrm{NaCl}, 2.5 \mathrm{KCl}, 1.6$ $\mathrm{CaCl}_{2}, 1.5 \mathrm{MgCl}_{2}, 1.25 \mathrm{NaH}_{2} \mathrm{PO}_{4}, 26 \mathrm{NaHCO}_{3}$, and 30 glucose (pH 7.4 after equilibration with $95 \% \mathrm{O}_{2}$ and $5 \% \mathrm{CO}_{2}$ ). For all recordings, slices were continuously superfused with oxygenated BBS, supplemented with the GABAa receptor blocker SR95531 (Gabazine; $2 \mu \mathrm{M}$ ) and with Tetrodotoxin (TTX; $500 \mathrm{nM}$ ), at $32-34{ }^{\circ} \mathrm{C}$. Electrophysiological recordings were performed from either dSPN dTomato positive, iSPN-dTomato negative, dSPN-GFP negative, or iSPN-GFP positive dorsal striatal cells. Cells were patched in the transmitted deep red light with which slices were visualized using a CoolSnap HQ CCD camera (Photometrics) run by Metamorph software (Universal Imaging) and mounted on either a Slicescope (Scientifica), or a BX51 (Olympus) microscope. Before patching, Tomato or GFP positive/negative cells were identified by the presence/absence of somatic fluorescence using LEDs of the corresponding excitation wavelengths (Thorlabs) coupled to the slice chamber via the epifluorescence pathway of the microscope. 
Pharmacology and Behavior analyses. The motor responses of Dlx5/6::Cre;Ebfi $f^{l /-}$ and Islet $1^{C r e /+} ; E b f f^{f l-}$ mice and their corresponding controls were examined in a round open field arena (diameter: $38 \mathrm{~cm}$ ) following injection of pharmacological agents. Minimum 2 weeks separated tests performed with different drugs on the same cohort of mice. For each condition, animals were acclimated to the experimental luminosity conditions (27-35 lux) of the test room for $1 \mathrm{~h}$. Following adaptation, baseline motor activity in the open field of each mouse was then measured for $8 \mathrm{~min}$ before subcutaneous injection with one of the following solutions: $0,9 \% \mathrm{NaCl}$ (control injection); $2 \mathrm{mg} / \mathrm{Kg}$ D1 receptor agonist SKF82958 (Sigma); and $1 \mathrm{mg} / \mathrm{Kg}$ D2 receptor agonist Quinpirole (Sigma). Drugs were administered in a volume of $10 \mathrm{ml} / \mathrm{Kg}$ of body weight. Following the injection, animals were left in their cage for $40 \mathrm{~min}$. They were then re-introduced into the arena for recording their motor activity over another 8 min-long timeperiod. Post hoc analysis of the total distance traveled was performed using Ethovision software (Noldus).

Statistical analyses. All data are presented as mean \pm SD or SEM (detailed in each figure legend). Two-tailed non-parametric Mann-Whitney $U$ test was used to compare two distributions in all experiments, with the exception of RNA-seq analysis (see above). All statistical analyses were performed using GraphPad Prism software. $p$-values are as follows: ${ }^{*} p<0.05,{ }^{* *} p<0.01,{ }^{* *} p<0.0001$.

\section{Data availability}

All relevant data are available within the article, supplementary files or available from the authors upon request. The raw RNA-seq data is deposited on GEO with accession code GSE120898.

Received: 11 April 2017 Accepted: 12 October 2018

Published online: 09 November 2018

\section{References}

1. Cui, G. et al. Concurrent activation of striatal direct and indirect pathways during action initiation. Nature 494, 238-242 (2013).

2. Freeze, B. S., Kravitz, A. V., Hammack, N., Berke, J. D. \& Kreitzer, A. C. Control of basal ganglia output by direct and indirect pathway projection neurons. J. Neurosci. 33, 18531-18539 (2013).

3. Kravitz, A. V., Tye, L. D. \& Kreitzer, A. C. Distinct roles for direct and indirect pathway striatal neurons in reinforcement. Nat. Neurosci. 15, 816-818 (2012).

4. Vicente, A. M., Galvão-Ferreira, P., Tecuapetla, F. \& Costa, R. M. Direct and indirect dorsolateral striatum pathways reinforce different action strategies. Curr. Biol. 26, R267-R269 (2016).

5. Gangarossa, G. et al. Spatial distribution of D1R- and D2R-expressing medium-sized spiny neurons differs along the rostro-caudal axis of the mouse dorsal striatum. Front. Neural Circuits 7, 124 (2013).

6. Yung, Kkl \& Bolam, Jp Localization of dopamine D1 and D2 receptors in the rat neostriatum: Synaptic interaction with glutamate- and GABA-containing axonal terminals. Synapse 38, 413-420 (2000).

7. Brimblecombe, K. R. \& Cragg, S. J. Substance P weights striatal dopamine transmission differently within the striosome-matrix axis. J. Neurosci. 35, 9017-9023 (2015).

8. Burguière, E., Monteiro, P., Mallet, L., Feng, G. \& Graybiel, A. M. Striatal circuits, habits, and implications for obsessive-compulsive disorder. Curr. Opin. Neurobiol. 30, 59-65 (2015).

9. Friedman, A. et al. A corticostriatal path targeting striosomes controls decision-making under conflict. Cell 161, 1320-1333 (2015).

10. Gerfen, C. R. The neostriatal mosaic: multiple levels of compartmental organization in the basal ganglia. Annu. Rev. Neurosci. 15, 285-320 (1992).

11. Smith, J. B. et al. Genetic-based dissection unveils the inputs and outputs of striatal patch and matrix compartments. Neuron 91, 1069-1084 (2016).

12. Halliday, A. L. \& Cepko, C. L. Generation and migration of cells in the developing striatum. Neuron 9, 15-26 (1992).

13. Hamasaki, T., Goto, S., Nishikawa, S. \& Ushio, Y. Neuronal cell migration for the developmental formation of the mammalian striatum. Brain. Res. Rev. 41, $1-12(2003)$.

14. Newman, H., Liu, F.-C. \& Graybiel, A. M. Dynamic ordering of early generated striatal cells destined to form the striosomal compartment of the striatum. J. Comp. Neurol. 523, 943-962 (2015).

15. Song, D. D. \& Harlan, R. E. Genesis and migration patterns of neurons forming the patch and matrix compartments of the rat striatum. Brain Res. Dev. Brain Res. 83, 233-245 (1994).

16. $\mathrm{Xu}, \mathrm{Z}$. et al. Sp8 and Sp9 coordinately promote $\mathrm{D} 2$-type medium spiny neuron production by activating Six 3 expression. Development 145, dev165456 (2018).
17. Kelly, S. M. et al. Radial glial lineage progression and differential intermediate progenitor amplification underlie striatal compartments and circuit organization. Neuron 99, 345-361.e4 (2018).

18. Arlotta, P., Molyneaux, B. J., Jabaudon, D., Yoshida, Y. \& Macklis, J. D. Ctip2 controls the differentiation of medium spiny neurons and the establishment of the cellular architecture of the striatum. J. Neurosci. 28, 622-632 (2008).

19. Ding, J. B., Oh, W.-J., Sabatini, B. L. \& Gu, C. Semaphorin 3E-plexinD1 signaling controls pathway-specific synapse formation in the striatum. Nat. Neurosci. 15, 215-223 (2011)

20. Long, J. E. et al. Dlx1\&2 and Mash1 transcription factors control striatal patterning and differentiation through parallel and overlapping pathways. $J$. Comp. Neurol. 512, 556-572 (2009).

21. Rataj-Baniowska, M. et al. Retinoic acid receptor $\beta$ controls development of striatonigral projection neurons through FGF-dependent and Meis1dependent mechanisms. J. Neurosci. 35, 14467-14475 (2015).

22. Wang, B., Waclaw, R. R., Allen, Z. J., Guillemot, F. \& Campbell, K. Ascll is a required downstream effector of Gsx gene function in the embryonic mouse telencephalon. Neural Dev. 4, 5 (2009).

23. Wang, B. et al. Loss of Gsx1 and Gsx2 function rescues distinct phenotypes in Dlx1/2 mutants. J. Comp. Neurol. 521, 1561-1584 (2013).

24. Ehrman, L. A. et al. The LIM homeobox gene Isll is required for the correct development of the striatonigral pathway in the mouse. Proc. Natl Acad. Sci. USA 110, E4026-E4035 (2013).

25. Garel, S., Marín, F., Grosschedl, R. \& Charnay, P. Ebf1 controls early cell differentiation in the embryonic striatum. Development 126, 5285-5294 (1999).

26. Lobo, M. K., Karsten, S. L., Gray, M., Geschwind, D. H. \& Yang, X. W. FACSarray profiling of striatal projection neuron subtypes in juvenile and adult mouse brains. Nat. Neurosci. 9, 443-452 (2006).

27. Lobo, M. K., Yeh, C. \& Yang, X. W. Pivotal role of early B-cell factor 1 in development of striatonigral medium spiny neurons in the matrix compartment. J. Neurosci. Res. 86, 2134-2146 (2008).

28. Lu, K.-M., Evans, S. M., Hirano, S. \& Liu, F.-C. Dual role for Islet-1 in promoting striatonigral and repressing striatopallidal genetic programs to specify striatonigral cell identity. Proc. Natl Acad. Sci. USA 111, E168-E177 (2014).

29. Zhang, Q. et al. The zinc finger transcription factor Sp9 is required for the development of striatopallidal projection neurons. Cell Rep. 16, 1431-1444 (2016).

30. Fishell, G. \& van der Kooy, D. Pattern formation in the striatum: neurons with early projections to the substantia nigra survive the cell death period. J. Comp. Neurol. 312, 33-42 (1991).

31. Hagimoto, K., Takami, S., Murakami, F. \& Tanabe, Y. Distinct migratory behaviors of striosome and matrix cells underlying the mosaic formation in the developing striatum. J. Comp. Neurol. 525, 794-817 (2017).

32. Krushel, L. A., Fishell, G. \& van der Kooy, D. Pattern formation in the mammalian forebrain: striatal patch and matrix neurons intermix prior to compartment formation. Eur. J. Neurosci. 7, 1210-1219 (1995).

33. Passante, L. et al. Temporal regulation of ephrin/Eph signalling is required for the spatial patterning of the mammalian striatum. Development 135, 3281-3290 (2008).

34. Gong, S. et al. A gene expression atlas of the central nervous system based on bacterial artificial chromosomes. Nature 425, 917-925 (2003).

35. Biezonski, D. K., Trifilieff, P., Meszaros, J., Javitch, J. A. \& Kellendonk, C. Evidence for limited D1 and D2 receptor co-expression and co-localization within the dorsal striatum of the neonatal mouse. J. Comp. Neurol. 523, 1175 (2015).

36. Morello, F. et al. Frizzled 3 controls axonal polarity and intermediate target entry during striatal pathway development. J. Neurosci. 35, 14205-14219 (2015).

37. Madisen, L. et al. A robust and high-throughput Cre reporting and characterization system for the whole mouse brain. Nat. Neurosci. 13, 133-140 (2010).

38. Fishell, G. \& van der Kooy, D. Pattern formation in the striatum: developmental changes in the distribution of striatonigral neurons. J. Neurosci. 7, 1969-1978 (1987).

39. Otsu, Y. et al. Optical monitoring of neuronal activity at high frame rate with a digital random-access multiphoton (RAMP) microscope. J. Neurosci. Methods 173, 259-270 (2008).

40. Elshatory, Y. \& Gan, L. The LIM-homeobox gene Islet-1 is required for the development of restricted forebrain cholinergic neurons. J. Neurosci. 28, 3291-3297 (2008).

41. Eisenstat, D. D. et al. DLX-1, DLX-2, and DLX-5 expression define distinct stages of basal forebrain differentiation. J. Comp. Neurol. 414, 217-237 (1999).

42. Ruest, L.-B., Hammer, R. E., Yanagisawa, M. \& Clouthier, D. E. Dlx5/6enhancer directed expression of Cre recombinase in the pharyngeal arches and brain. Genesis 37, 188-194 (2003). 
43. Srinivas, S. et al. Cre reporter strains produced by targeted insertion of EYFP and ECFP into the ROSA26 locus. BMC Dev. Biol. 1, 4 (2001).

44. Zerucha, T. et al. A highly conserved enhancer in the Dlx5/Dlx6Intergenic region is the site of cross-regulatory interactions betweenDlx genes in the embryonic forebrain. J. Neurosci. 20, 709-721 (2000).

45. Lee, S. et al. STAT3 promotes motor neuron differentiation by collaborating with motor neuron-specific LIM complex. Proc. Natl Acad. Sci. USA 110, 11445-11450 (2013).

46. Gokce, O. et al. Cellular taxonomy of the mouse striatum as revealed by single-cell RNA-seq. Cell Rep. 16, 1126-1137 (2016).

47. Chen, Y.-C. et al. Foxp2 controls synaptic wiring of corticostriatal circuits and vocal communication by opposing Mef2c. Nat. Neurosci. 19, 1513-1522 (2016).

48. Mega, T. et al. Zinc finger protein 521 antagonizes early B-cell factor 1 and modulates the B-lymphoid differentiation of primary hematopoietic progenitors. Cell Cycle 10, 2129-2139 (2011).

49. Zhang, Z. et al. Mutation of SLC35D3 causes metabolic syndrome by impairing dopamine signaling in striatal D1 neurons. PLoS Genet. 10, e1004124 (2014).

50. Ehrlich, M. E., Rosen, N. L., Kurihara, T., Shalaby, I. A. \& Greengard, P. DARPP-32 development in the caudate nucleus is independent of afferent input from the substantia nigra. Brain Res. Dev. Brain Res. 54, 257-263 (1990).

51. Markowitz, J. E. et al. The striatum organizes 3D behavior via moment-tomoment action selection. Cell 174, 44-58.e17 (2018).

52. Faedo, A. et al. Differentiation of human telencephalic progenitor cells into MSNs by inducible expression of Gsx2 and Ebf1. Proc. Natl Acad. Sci. USA 114, E1234-E1242 (2017).

53. Marin, O., Anderson, S. A. \& Rubenstein, J. L. Origin and molecular specification of striatal interneurons. J. Neurosci. 20, 6063-6076 (2000).

54. Dodson, P. D. et al. Distinct developmental origins manifest in the specialized encoding of movement by adult neurons of the external globus pallidus. Neuron 86, 501-513 (2015).

55. Nóbrega-Pereira, S. et al. Origin and molecular specification of globus pallidus neurons. J. Neurosci. 30, 2824-2834 (2010)

56. Bielle, F. et al. Slit 2 activity in the migration of guidepost neurons shapes thalamic projections during development and evolution. Neuron 69, 1085-1098 (2011).

57. Tinterri, A. et al. Tangential migration of corridor guidepost neurons contributes to anxiety circuits. J. Comp. Neurol. 526, 397-411 (2018).

58. Waclaw, R. R., Ehrman, L. A., Pierani, A. \& Campbell, K. Developmental origin of the neuronal subtypes that comprise the amygdalar fear circuit in the mouse. J. Neurosci. 30, 6944-6953 (2010).

59. Bupesh, M., Abellán, A. \& Medina, L. Genetic and experimental evidence supports the continuum of the central extended amygdala and a mutiple embryonic origin of its principal neurons. J. Comp. Neurol. 519, 3507-3531 (2011).

60. Nadarajah, B. \& Parnavelas, J. G. Modes of neuronal migration in the developing cerebral cortex. Nat. Rev. Neurosci. 3, 423-432 (2002).

61. Boller, S. \& Grosschedl, R. The regulatory network of B-cell differentiation: a focused view of early B-cell factor 1 function. Immunol. Rev. 261, 102-115 (2014).

62. Gyory, I. et al. Transcription factor Ebf1 regulates differentiation stage-specific signaling, proliferation, and survival of B cells. Genes Dev. 26, 668-682 (2012).

63. Tursun, B., Patel, T., Kratsios, P. \& Hobert, O. Direct conversion of C. elegans germ cells into specific neuron types. Science 331, 304-308 (2011).

64. Boller, S. et al. Pioneering activity of the C-terminal domain of EBF1 shapes the chromatin landscape for B cell programming. Immunity 44, 527-541 (2016).

65. Guilhamon, P. et al. Meta-analysis of IDH-mutant cancers identifies EBF1 as an interaction partner for TET2. Nat. Commun. 4, 2166 (2013).

66. Lin, H. \& Grosschedl, R. Failure of B-cell differentiation in mice lacking the transcription factor EBF. Nature 376, 263-267 (1995).

67. Lokmane, L. et al. Sensory map transfer to the neocortex relies on pretarget ordering of thalamic axons. Curr. Biol. 23, 810-816 (2013).
68. Xu, Q., Tam, M. \& Anderson, S. A. Fate mapping Nkx2.1-lineage cells in the mouse telencephalon. J. Comp. Neurol. 506, 16-29 (2008).

69. Espinosa-Medina, I. et al. The sacral autonomic outflow is sympathetic. Science 354, 893-897 (2016)

70. Anders, S. \& Huber, W. Differential expression analysis for sequence count data. Genome Biol. 11, R106 (2010).

\section{Acknowledgements}

We thank members of the S.G. lab, M. Casado, J.A. Girault, C. Goridis, D. Hervé, C Lena, and E. Valjent for stimulating discussions. We are grateful to C. Auger, D. Souchet, A. Boudjouher, and C. Le Moal for excellent technical assistance. We thank A. de Kerchove, E. Thierion, and L. Danglot for scientific input. We are grateful to the IBENS Imaging Facility (France BioImaging, supported by ANR-10-INBS-04, ANR-10-LABX54 MEMO LIFE and ANR-11-IDEX-000-02 PSL* Reseach University, "Investments for the future"; NERF N²011-45; FRM DGE 20111123023; and FRC Rotary International France), the IBENS Genomics Facility (supported by the France Génomique national infrastructure, funded as part of the "Investissements d'Avenir" program managed by the Agence Nationale de la Recherche, ANR-10-INBS-09) and the IBENS acute transgenesis facility. A.T. is part of the ENP doctoral program. A.T. was supported by a doctoral fellowship from Boehringer Ingelheim Fonds (BIF), the ENP program and the Labex Memolife «Investements for the future » (ANR-10-LABX-54 MEMO LIFE and ANR-11 IDEX-0001-02 PSL $^{*}$ Research University). This work was supported by grants from INSERM, CNRS, the ANR program (ANR-12-BVS4-0010-01) and the ERC NImO (616080) to S.G, from the ANR program (ANR-12-JSV4-004) to D.P. and from NIH (R01 MH090740) to K.C.

\section{Author contributions}

A.T. and S.G. designed the study and wrote the manuscript. M.A.D. performed and analyzed electrophysiological recordings. M.A.D., F.M., D.P., and A.T. performed motor behavior experiments and analyses. F.C. and S.L. performed the RNA-seq and transcriptomic analyses. BM provided expertise for two-photon imaging. A.T., L.L., C.M., and M.K. performed all other experiments and analyzed data. I.G., P.M.S., K.C., and R.G. provided mouse models. All the authors edited the manuscript. S.G. supervised the project.

\section{Additional information}

Supplementary Information accompanies this paper at https://doi.org/10.1038/s41467 018-07171-4.

Competing interests: The authors declare no competing interests.

Reprints and permission information is available online at http://npg.nature.com/ reprintsandpermissions/

Publisher's note: Springer Nature remains neutral with regard to jurisdictional claims in published maps and institutional affiliations.

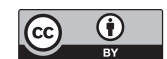

Open Access This article is licensed under a Creative Commons Attribution 4.0 International License, which permits use, sharing, adaptation, distribution and reproduction in any medium or format, as long as you give appropriate credit to the original author(s) and the source, provide a link to the Creative Commons license, and indicate if changes were made. The images or other third party material in this article are included in the article's Creative Commons license, unless indicated otherwise in a credit line to the material. If material is not included in the article's Creative Commons license and your intended use is not permitted by statutory regulation or exceeds the permitted use, you will need to obtain permission directly from the copyright holder. To view a copy of this license, visit http://creativecommons.org/ licenses/by/4.0/.

(C) The Author(s) 2018 